\title{
Sliding friction across the scales: Thermomechanical interactions and dissipation partitioning
}

\author{
İ. Temizer \\ Department of Mechanical Engineering, Bilkent University, 06800 Ankara, Turkey
}

\section{A R T I C L E I N F O}

\section{Article history:}

Received 14 December 2014

Received in revised form

4 September 2015

Accepted 19 January 2016

Available online 29 January 2016

Keywords:

Sliding friction

Dissipation

Contact homogenization

Thermo-viscoelasticity

\begin{abstract}
A B S T R A C T
A homogenization framework is developed for determining the complete macroscopic thermomechanical sliding contact response of soft interfaces with microscopic roughness. To this end, a micro-macro mechanical dissipation equality is first established which enables defining a macroscopic frictional traction. The derivation allows both contacting bodies to be deformable, thereby extending the commonly adopted setting where one of the bodies is rigid. Moreover, it forms a basis for the second step, where a novel micromacro thermal dissipation equality is established which enables defining partitioning coefficients that are associated with the frictional dissipation as it is perceived on the macroscale. Finally, a comparison of the temperature fields from the original heterogeneous thermomechanical contact problem and an idealized homogeneous one reveals an identification of the macroscopic temperature jump. The computational implementation of the framework is carried out within an incrementally two-phase micromechanical test which delivers a well-defined macroscopic response that is not influenced by purely algorithmic choices such as the duration of sliding. Two-dimensional numerical investigations on periodic and random samples from thermo-viscoelastic boundary layers with unilateral and bilateral roughness demonstrate the temperature-velocity-pressure dependence of the macroscopic contact response.
\end{abstract}

(c) 2016 Elsevier Ltd. All rights reserved.

\section{Introduction}

The multiscale nature of friction has long been recognized (Bowden and Tabor, 2008; Persson, 2000). Friction appears in one form or another at the nano-micro-macro scales and in all cases it represents irreversible energy transfer away from the contact zone, in other words dissipation. The goal of this work is to develop a theoretical and computational framework for characterizing how microscopic dissipation accumulates across multiple length scales and manifests itself as sliding friction on an upper scale for soft materials. The differentiating aspect of the work with respect to earlier contributions is twofold: first, both bodies in contact are admitted to be finitely deformable, and second, the aim is to take multiscale thermomechanical interactions fully into account. Overall, the emphasis of the framework will be not only on mechanical contact quantities of interest, such as a friction coefficient, but also on thermal ones, such as how dissipation - as it is perceived on the macroscale - is partitioned onto the contacting surfaces.

Presently, the focus is on the continuum-level modeling of soft interfaces, which are typically associated with biological or synthetic materials. Recently, there has been significant interest in the modeling of tactile perception which requires the

E-mail address: temizer@bilkent.edu.tr 
frictional characterization between skin and textured surfaces or textile materials (Fagiani et al., 2012; van Kuilenburg et al., 2012; Adams et al., 2013; Koç and Aksu, 2013; Ramalho et al., 2013). There has also been interest in the frictional response of compliant engineered surfaces and how these surfaces interact with relatively hard rough or textured surfaces (Shen et al., 2008; Murarash et al., 2011; Brörmann et al., 2013; Bai et al., 2014), rubber being a particular example of technological interest (Le Gal and Klüppel, 2008; Carbone et al., 2009). For such soft materials, a major source of dissipation is the viscoelastic response of the material. The boundary layer in the vicinity of contact zone is continuously and cyclically being loaded by the asperities of the rough surface during sliding, which causes dissipation. Indeed, starting with the early works by Schallamach (1953), Grosch (1963) and others, early models of rubber friction already recognized viscous dissipation as one of the two major contributions, the other being adhesion. While significant advances have been achieved towards a quantitative modeling of rubber friction (Persson et al., 2005), thereby constituting a basis for similar studies for soft materials in general, emphasis has predominantly been on the mechanical aspects that can be concatenated towards a friction coefficient value which depends on a number of contact variables including the pressure and the slip velocity. Studies towards this purpose have been carried out in both infinitesimal (Klüppel and Heinrich, 2000; Persson, 2001; Carbone and Putignano, 2013) and finite deformation regimes (Wriggers and Reinelt, 2009; Temizer and Wriggers, 2010a; De Lorenzis and Wriggers, 2013), all being based on a setting where one surface is smooth and deformable while the other is rough and rigid. On the other hand, from everyday experience one is familiar with how rapidly soft materials can warm up due to friction and how both surfaces can significantly deform, for instance when hands are rubbed against each other. Since viscous response is highly temperature sensitive, the thermal aspects of the problem are just as important as the mechanical ones (Persson, 2014). The macroscopic investigation of these thermal aspects requires more than the temperature dependence characterization of friction. For instance, the microscopically rough interface topography induces a temperature jump across the macroscopic contact interface which influences the temperature distribution in the vicinity of the contact interface (Madhusudana, 1996). More importantly, temperature changes are also governed by how the frictional dissipation is partitioned onto the contacting surfaces as heat flux on the macroscale. On a phenomenological level, a fraction of the mechanical power dissipated is absorbed by one of the surfaces and the remaining by the other (Agelet de Saracibar, 1999). Hence, unless the nature of this partitioning is described accurately, the macroscopic temperature rise and hence its overall impact on friction cannot be appropriately assessed. A major objective in this work will be to provide a non-phenomenological basis for macroscopic frictional dissipation partitioning onto deformable surfaces in sliding contact. Efforts towards this purpose have so far been limited to one-dimensional models that concentrate only on the thermal aspects of the problem (Chantrenne and Raynaud, 1997, 2001; Nosko, 2013).

The microscopic dissipation mechanisms include bulk and interface dissipation. Here, the bulk dissipation will be specialized to soft materials. As representative recoverable and irrecoverable deformation mechanisms, viscoelasticity and damage are considered. While plasticity can also be observed for the materials of interest, in particular for rubber-like materials (Lion, 1997; Miehe and Keck, 2000), the vanishing dissipation contribution due to such irrecoverable mechanisms can be demonstrated through damage alone. Interface dissipation will be limited to microscopic friction while adhesion, which can have a significant contribution to macroscopic friction (Persson et al., 2005; Momozono et al., 2012), is omitted. Nevertheless, adhesive effects can be incorporated through an identical treatment. Despite their significance towards a quantitative understanding of friction, a number of additional simplifications will be admitted in view of the fact that these do not affect the qualitative conclusions to be drawn in this work. These include the omission of inertial effects (Momozono et al., 2012; Bai et al., 2014), the assumption of a single scale of roughness versus a fractal structure (Persson et al., 2005; Pei et al., 2005) and the common choice of a single relaxation time instead of employing a sufficiently large number to capture the relaxation spectrum (Wriggers and Reinelt, 2009; Suwannachit et al., 2012; Carbone and Putignano, 2013).

The work is outlined as follows. In Section 2, the continuum mechanics background for the thermomechanical contact problem is summarized. The presentation here is brief in order to allow a rapid transition to the major discussion yet provides a sufficiently general basis for it. The micro-macro thermal dissipation equality that is central to this work is established in Section 3, based on the statement of its mechanical counterpart in a form that is suitable for two deformable micromechanical samples in contact. Specifically, the partitioning coefficients that are associated with the frictional dissipation as it is perceived on the macroscale will be identified in this section. Subsequently, an incrementally two-phase micromechanical test will be described in Section 4 where the determination of the temperature jump across the macroscopic contact interface will additionally be discussed. Here, the explicit enforcement of the macroscopic contact surface temperatures will be the key to ensuring the algorithmic consistency of the overall homogenization framework, in addition to its thermodynamic consistency that is guaranteed by the micro-macro dissipation equalities. The thermo-viscoelastic material model employed will be outlined in Section 5, which will also enable the verification of various assumptions that were invoked earlier. In Section 6, two-dimensional numerical investigations on periodic/random samples from thermoviscoelastic boundary layers with unilateral/bilateral roughness will demonstrate the temperature-velocity-pressure dependence of the macroscopic contact response. Finally, concluding remarks address a number of simplifications that have been assumed throughout the work. Specific choices regarding constitutive modeling and the simulation parameters are provided in the appendices. 


\section{Continuum framework}

In this section, following standard texts (Holzapfel, 2001; Gurtin et al., 2010), the continuum framework for a thermomechanical contact problem is outlined in as general a setting as possible for the purposes of the present study. A modeling framework for isotropic thermo-viscoelastic materials in frictional contact will be outlined in Section 5. Specific constitutive modeling choices will be stated in Appendix A.

\subsection{Thermo-inelasticity}

The linear momentum balance for a finitely deforming medium is

$$
\operatorname{Div}[\boldsymbol{P}]+\rho_{0} \boldsymbol{b}=\rho_{0} \ddot{\boldsymbol{x}}
$$

where $\boldsymbol{P}$ is the first Piola-Kirchhoff stress tensor, $\boldsymbol{b}$ is the body force and $\rho_{\mathrm{o}}$ is the referential density. The divergence and gradient operations are with respect to the reference configuration $\mathcal{R}_{0}$ with position vector $\boldsymbol{X}$ such that the deformation gradient tensor is $\boldsymbol{F}=\operatorname{Grad}[\boldsymbol{x}]$ where $\boldsymbol{x}$ is the position vector in the current configuration $\mathcal{R}$. Per unit volume of $\mathcal{R}_{0}$, the thermodynamic potentials $e$ (internal energy), $\eta$ (entropy) and $\psi$ (Helmholtz energy) are introduced where $\psi=e-\theta \eta$ is a canonical function of $\boldsymbol{F}$, temperature $\theta$ and a set of internal variables $\mathcal{I}$ reflecting inelasticity. Under standard assumptions, the second law induces relations among thermomechanical quantities as well as the non-negativity of the mechanical $(\mathcal{M})$ and thermal $(\mathcal{T})$ contributions to dissipation:

$$
\boldsymbol{P}=\frac{\partial \psi}{\partial \boldsymbol{F}}, \quad \eta=-\frac{\partial \psi}{\partial \theta}, \quad \mathcal{M}=\mathcal{A} \cdot \dot{I} \geq 0, \quad \mathcal{T}=-\boldsymbol{Q} \cdot \boldsymbol{G} / \theta \geq 0
$$

Here, $\boldsymbol{G}=\operatorname{Grad}[\theta], \boldsymbol{Q}$ is the referential heat flux vector and $\mathcal{A}=-\partial \psi / \partial \boldsymbol{I}$ is the thermodynamic driving force for $\mathcal{I}$. Defining a heating term

$$
\mathcal{H}=-\theta \frac{\partial \boldsymbol{P}}{\partial \theta} \cdot \dot{\boldsymbol{F}}+\theta \frac{\partial \mathcal{A}}{\partial \theta} \cdot \dot{\mathcal{I}}
$$

the energy balance can now be stated in the form (cf. Simo et al., 1998, Section 57)

$$
c \dot{\theta}=\mathcal{M}-\mathcal{H}-\operatorname{Div}[\mathbf{Q}]+\rho_{0} r
$$

where $r$ is the heat supply and $c=\partial e / \partial \theta=\theta \partial \eta / \partial \theta=-\theta \partial^{2} \psi / \partial \theta^{2}$ is the heat capacity. Also, taking $c$ as the starting point (Chadwick and Creasy, 1984; Reese and Govindjee, 1998a), the general form for $\psi$ may be expressed as $\left(\vartheta=\theta-\theta_{0}\right)$

$$
\psi(\boldsymbol{F}, \theta, \mathcal{I})=\frac{\theta}{\theta_{o}} \psi_{o}(\boldsymbol{F}, \mathcal{I})-\frac{\vartheta}{\theta_{o}} e_{o}(\boldsymbol{F}, \mathcal{I})+\int_{\theta_{o}}^{\theta}\left(1-\frac{\theta}{\theta^{\prime}}\right) c\left(\boldsymbol{F}, \theta^{\prime}, \mathcal{I}\right) \mathrm{d} \theta^{\prime} .
$$

Here, $\psi_{0}$ and $e_{o}$ indicate the material characterization at a reference temperature $\theta_{0}$ while the last integral embodies the complexities of temperature dependence via c. $\psi$ must be supplemented by a set of evolution laws for $\mathcal{I}$, typically stated in terms of $\mathcal{A}$, and a conduction model $\boldsymbol{Q}(\boldsymbol{F}, \boldsymbol{G}, \boldsymbol{\theta}, \mathcal{I})$ that satisfy, respectively, $(2.2)_{3}$ and $(2.2)_{4}$, all additionally being subject to frame invariance.

\subsection{Contact interfaces}

Denoting the outward unit normal to the boundary $\partial \mathcal{R}$ by $\boldsymbol{n}$,

$$
\boldsymbol{t}=\boldsymbol{T n}, \quad h=-\boldsymbol{q} \cdot \boldsymbol{n}
$$

must be additionally specified as boundary conditions (BCs) where $\boldsymbol{T}$ is the Cauchy stress and $\boldsymbol{q}$ is the spatial heat flux. In order to treat contact, following standard approaches (Laursen, 2003; Wriggers, 2006) but without concern for numerical details, one of the bodies in contact is designated as slave $\left(\mathcal{R}^{(1)}\right)$ and the other as master $\left(\mathcal{R}^{(2)}\right)$. At the contact interface $\Gamma^{\mathrm{c}}$, the contact interactions imply

$$
\boldsymbol{t}^{(1)}+\boldsymbol{t}^{(2)}=\mathbf{0}, \quad h^{(1)}+h^{(2)}=\mathcal{F}
$$

where $\mathcal{F}$ is the frictional dissipation per unit area of $\Gamma^{c}$ in case of slip. For notational compactness, traction variables without a superscript will be associated with the slave following Laursen (2003). The contact tractions may then be decomposed into normal and tangential parts as

$$
\boldsymbol{t}^{(1)}=\boldsymbol{t}=\boldsymbol{t}_{N}+\boldsymbol{t}_{T}, \quad \boldsymbol{t}^{(2)}=-\boldsymbol{t} .
$$

Endowing the master surface with convected curvilinear coordinates $\xi^{\alpha}(\alpha=1,2)$, one may write

$$
\boldsymbol{t}_{N}=t_{N} \boldsymbol{n}^{(2)}, \quad \boldsymbol{t}_{T}=-t_{T \alpha} \boldsymbol{a}^{\alpha}
$$


where $t_{N}$ is the contact pressure and $\boldsymbol{a}^{\alpha}\left(\boldsymbol{a}_{\alpha}\right)$ are the contravariant (covariant) tangential vectors on $\partial \mathcal{R}^{(2)}$ induced by $\xi^{\alpha}$. With this convention, the frictional dissipation takes the form

$$
\mathcal{F}=-\boldsymbol{t}_{T} \cdot \boldsymbol{v}_{T}=t_{T \alpha} \dot{\xi}^{\alpha} \geq 0
$$

where $\boldsymbol{v}_{T}=\dot{\xi}^{\beta} \boldsymbol{a}_{\beta}$ is the slip velocity. The contact heat flux may now be explicitly decomposed as

$$
h^{(1)}=h^{c}+\gamma^{(1)} \mathcal{F}, \quad h^{(2)}=-h^{c}+\gamma^{(2)} \mathcal{F}
$$

where $\gamma^{(I)}$, relative effusivities (Agelet de Saracibar, 1999), are contact constitutive variables which control the partitioning of the frictional dissipation onto the contacting surfaces. Due to $(2.7)_{2}$, they are subject to

$$
\gamma^{(1)}+\gamma^{(2)}=1
$$

The conduction part $h^{c}$ of the contact heat flux may be prescribed via two different models. In the first model, motivated by microscopic contact mechanisms (Madhusudana, 1996; Bahrami et al., 2006), it is constitutively specified such that a finite temperature jump $\vartheta_{c}$ is observed at the interface due to contact resistance:

$$
\vartheta_{c}=\theta^{(1)}-\theta^{(2)}
$$

In the second model, for well-conforming surfaces as well as for theoretical purposes, it is advantageous to consider a constraint scenario where, analogous to a vanishing gap, contacting surfaces pick the same temperature: $\vartheta_{c}=0$. The enforcement of this constraint is based on methods for the enforcement of non-penetration and stick conditions. For either scenario,

$$
h^{c}=\frac{\partial \mathcal{H}^{c}}{\partial \vartheta_{c}}
$$

delivers the flux from the interface dissipation potential $\mathcal{H}^{c}$ which is primarily a function of $\vartheta_{c}$ but is also dependent on microstructural changes of the interface topography (Temizer and Wriggers, 2010b). For the second model, this relation is motivated by a penalty-regularization of the constraint and constitutes a basis for alternative numerical schemes of constraint enforcement - see Section 5.2.

It is possible to pursue a more general thermal contact model that assigns the contact interface a temperature that is independent of the slave/master surfaces (Strömberg et al., 1996; Agelet de Saracibar, 1999). However, this model condenses to the summarized one whenever the energy stored by the independent interface is negligible (see Laursen, 2003, p. 258), an assumption that is also consistent with the homogenization approach to be pursued.

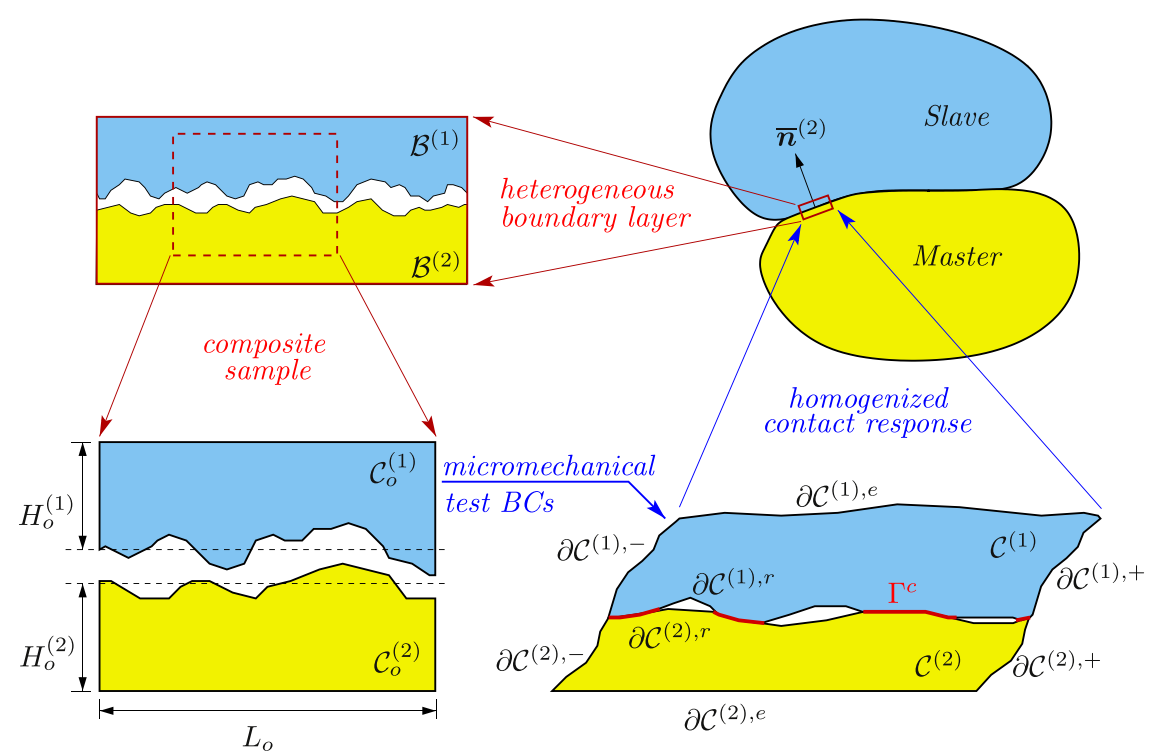

Fig. 1. The heterogeneous contact problem described in Section 3.1.1 is depicted. The composite sample $C_{0}=C_{0}^{(1)} \cup C_{0}^{(2)}$ from the heterogeneous boundary layer is subjected to the BCs outlined in Section 3.1.2 in order to obtain the homogenized contact response at a macroscopic contact point. While not explicitly shown, the length of the samples after deformation will be denoted by $L$. See Figs. 4 and 5 for simulation instances. 


\section{Space-time contact homogenization}

Within the framework summarized in Section 2 that is sufficiently general to adequately model a broad range of problems, the multiscale thermomechanical modeling of sliding friction will be carried out, considering both the linear momentum and the energy balance. For this purpose, the geometry of the micromechanical sample is summarized next, based on the ideas presented in Stupkiewicz (2007).

\subsection{Micromechanical testing procedure}

\subsubsection{Test geometry}

The micromechanical testing procedure represents the approach used in the extraction of the homogenized response. The geometry and the test are depicted in Fig. 1. Macroscopically, the boundary layers $\mathcal{B}^{(1)}$ and $\mathcal{B}^{(2)}$ of the two bodies come into contact. The boundary layers are assumed to be homogeneous within the bulk but heterogeneous on the surface due to roughness. Their macroscopic curvatures are not reflected to the microscale since a scale separation assumption will be continuously employed throughout this work. From $\mathcal{B}^{(I)}$, an initially matching cut is made to obtain a composite sample, constituting individual sample domains $C_{o}^{(I)}$ in the reference configuration. In subsequent discussions, the domain or boundary without the subscript is associated with the composite sample and the omission of the subscript refers to the current configuration, e.g. $C_{0}=C_{0}^{(1)} \cup C_{0}^{(2)}$ and $\partial C=\partial C^{(1)} \cup \partial C^{(2)}$.

The heights $H_{o}^{(I)}$ of the samples are arbitrarily large, although in practice one wishes to choose them as small as possible for numerical efficiency. The lengths $L_{0}^{(1)}=L_{0}^{(2)}=L_{0}$ are chosen sufficiently large to ensure that the surface roughness is statistically well-represented, the determination of which is non-trivial once either surface is assigned a non-periodic roughness. When both are periodic, it is often straightforward to determine $L_{o}$ based on the identification of a composite unit cell. In a general numerical setting with randomness, $H_{o}^{(I)}$ and $L_{o}$ are incrementally increased to incorporate thicker and wider portions of the boundary layers until the monitored macroscopic response is rendered insensitive to further changes. Such a composite sample constitutes a representative contact element (RCE) (Temizer and Wriggers, 2008).

\subsubsection{Boundary conditions}

The boundaries $\partial C_{0}^{(I)}$ are divided into three major portions where both (i) appropriate BCs are enforced in order to project down selected contact variables from the macro- to the microscale and (ii) variables dual to the enforced ones are determined and projected back up through space-time averaging. The latter part is discussed in Sections 3.2 and 3.3. The three portions are:

1. $\partial C_{0}^{(I), i}$ - Interior Boundaries: These coincide with the cutting plane of $C^{(I)}$ from $\mathcal{B}^{(I)}$, decomposed into opposing $+/$ - surfaces $C^{(l),+} \cup C^{(l),-}=C^{(I), i}$ where periodic BCs are applied, enforcing periodic temperature/displacement and anti-periodic traction/flux fields.

2. $\partial C_{0}^{(I), r}$ - Rough Boundaries: The rough surfaces with a prescribed root-mean-square (RMS) roughness $\sigma^{(I)}$ constitute the potential contact domain. The contact traction and heat flux is non-zero only on the contact portion $\Gamma^{\mathrm{c}}$ of $\partial C^{(I), r}$ where computational contact mechanics methods are applied - see Section 5.2.

3. $\partial C_{o}^{(I), e}$ - Exterior Boundaries: These constitute the observable surfaces of the composite sample where an experimental control over the test conditions would be possible and measurements would be carried out. Here, the macroscopic tangential velocities $\overline{\boldsymbol{v}}_{T}^{(I)}$ of the contacting points are enforced. Additionally, the macroscopic normal velocity $\bar{v}_{N}$ is enforced on $\partial C_{o}^{(2), e}$ while the macroscopic pressure $\bar{t}_{N}$ is enforced on $\partial C_{0}^{(1), e}$.

The thermal BCs on $\partial C_{o}^{(I), e}$, presently assumed to be temperature controlled without loss of generality, have a strong influence on the conductive macroscopic heat flux $\bar{h}^{c}$ and will be discussed in Section 4. Additionally, the relative motion between the two samples requires periodicity conditions on the geometrical configuration. When both samples are periodic, it is natural to impose such conditions by checking the contact between the slave unit cell surface $C^{(1), e}$ not only with the original master unit cell surface $C^{(2), e}$ but also with all of its images. A similar procedure is applied when either of the surfaces displays random roughness. Finally, Fig. 1 reflects the deformation of the composite sample due to the projection of the macroscopic surface stretch, i.e. the deformation of the boundary layer in the plane of macroscopic contact such that the edge length changes from $L_{o}$ to $L$, although this effect will be omitted in the numerical examples to be presented - cf. Sadowski and Stupkiewicz (2010), Temizer and Wriggers (2010b), and McBride et al. (2012).

\subsection{Mechanical contact homogenization}

For the space-time homogenization of the mechanical contact interactions, the approach pursued in Temizer and Wriggers (2010a) is generalized to account for the contact of two deformable boundary layers. The mechanical case will also constitute a basis for carrying out the thermal contact homogenization. Defining $\boldsymbol{v}=\dot{\boldsymbol{x}}$, the starting point will be the integral form of $(2.1)$ : 


$$
\int_{C_{o}} \boldsymbol{P} \cdot \dot{\boldsymbol{F}} \mathrm{d} V=\int_{\partial C} \boldsymbol{t} \cdot \boldsymbol{v} \mathrm{d} a+\int_{C_{o}} \rho_{o}(\boldsymbol{b}-\ddot{\boldsymbol{x}}) \cdot \boldsymbol{v} \mathrm{d} V
$$

\subsubsection{Stress power}

The stress power contribution to the left-hand side of (3.1) can be expanded as

$$
\boldsymbol{P} \cdot \dot{\boldsymbol{F}}=\dot{\psi}+\mathcal{M}+\eta \dot{\theta}=\dot{\psi}+\eta \dot{\theta}+\mathcal{D}+\mathcal{V}
$$

where $\mathcal{M}$ has been decomposed into damage $(\mathcal{D})$ and viscous $(\mathcal{V})$ parts as representative irrecoverable and recoverable inelastic behaviors - see Appendix A. For the viscous part, the surface-projected averages

$$
\left\langle\mathcal{V}^{(I)}\right\rangle=\frac{1}{\mathcal{A}} \int_{C_{0}^{(I)}} \mathcal{V} \mathrm{d} V
$$

are additionally introduced, leading to the expression

$$
\frac{1}{\mathcal{A}} \int_{C_{0}} \boldsymbol{P} \cdot \dot{\boldsymbol{F}} \mathrm{d} V=\left\langle\mathcal{V}^{(1)}\right\rangle+\left\langle\mathcal{V}^{(2)}\right\rangle+\frac{1}{\mathcal{A}} \int_{C_{0}}(\dot{\psi}+\eta \dot{\theta}+\mathcal{D}) \mathrm{d} V .
$$

Above, $\mathcal{A}$ is the projected area of $\partial C^{r}$, e.g. equal to $L$ in Fig. 1 .

\subsubsection{Non-observable surfaces}

The traction contribution to the right-hand side of (3.1) is

$$
\int_{\partial C} \boldsymbol{t} \cdot \boldsymbol{v} \mathrm{d} a=\int_{\partial C^{i}} \boldsymbol{t} \cdot \boldsymbol{v} \mathrm{d} a+\int_{\partial C^{r}} \boldsymbol{t} \cdot \boldsymbol{v} \mathrm{d} a+\int_{\partial C^{e}} \boldsymbol{t} \cdot \boldsymbol{v} \mathrm{d} a
$$

where the integral over the inner surfaces $\partial C^{i}$ vanishes due to periodic BCs. The integral in (3.5) on the rough interface $\partial C^{r}$ is non-zero only on the contact interface $\Gamma^{\mathrm{c}}$ (Laursen, 2003; Wriggers, 2006):

$$
\int_{\partial C^{r}} \boldsymbol{t} \cdot \boldsymbol{v} \mathrm{d} a=\int_{\Gamma^{c}}\left(\boldsymbol{t}^{(1)} \cdot \boldsymbol{v}^{(1)}+\boldsymbol{t}^{(2)} \cdot \boldsymbol{v}^{(2)}\right) \mathrm{d} a=\int_{\Gamma^{c}} \boldsymbol{t} \cdot\left(\boldsymbol{v}^{(1)}-\boldsymbol{v}^{(2)}\right) \mathrm{d} a=-\int_{\Gamma^{c}} \mathcal{F} \mathrm{d} a .
$$

Defining a surface average $\langle\mathcal{F}\rangle$, one obtains

$$
\langle\mathcal{F}\rangle=\frac{1}{\mathcal{A}} \int_{\Gamma^{c}} \mathcal{F} \mathrm{d} a \rightarrow \frac{1}{\mathcal{A}} \int_{\partial C^{r}} \boldsymbol{t} \cdot \boldsymbol{v} \mathrm{d} a=-\langle\mathcal{F}\rangle .
$$

\subsubsection{Observable surfaces}

A microscopic vector $\boldsymbol{a}$ may be decomposed into its perpendicular $(\perp)$ and parallel $(\|)$ components with respect to the normal $\overline{\boldsymbol{n}}^{(2)}$ of the macroscopic master surface (see Fig. 1):

$$
a_{\perp}=\boldsymbol{a} \cdot \overline{\boldsymbol{n}}^{(2)}, \quad \boldsymbol{a}_{\|}=\boldsymbol{a}-a_{\perp} \overline{\boldsymbol{n}}^{(2)}
$$

The last integral in (3.5) over the exterior observation surfaces is then

$$
\begin{aligned}
\int_{\partial C^{e}} \boldsymbol{t} \cdot \boldsymbol{v} \mathrm{d} a & =\int_{\partial C^{(1), e}} \boldsymbol{t} \cdot \boldsymbol{v} \mathrm{d} a+\int_{\partial C^{(2), e}} \boldsymbol{t} \cdot \boldsymbol{v} \mathrm{d} a=\int_{\partial C^{(1), e}}\left(t_{\perp} v_{\perp}+\boldsymbol{t}_{\|} \cdot \boldsymbol{v}_{\|}\right) \mathrm{d} a+\int_{\partial C^{(2), e}}\left(t_{\perp} v_{\perp}+\boldsymbol{t}_{\|} \cdot \boldsymbol{v}_{\|}\right) \mathrm{d} a \\
& =\int_{\partial C^{(1), e}}\left(-\bar{t}_{N} v_{\perp}+\boldsymbol{t}_{\|} \overline{\boldsymbol{v}}_{T}^{(1)}\right) \mathrm{d} a+\int_{\partial C^{(2), e}}\left(t_{\perp} \bar{v}_{N}+\boldsymbol{t}_{\|} \overline{\boldsymbol{v}}_{T}^{(2)}\right) \mathrm{d} a
\end{aligned}
$$

after making use of the BCs on $\partial C^{(I), e}$ (Section 3.1.2). The surface averages

$$
\left\langle v_{\perp}^{(1)}\right\rangle_{e}=\frac{1}{\mathcal{A}} \int_{\partial C^{(1), e}} v_{\perp} \mathrm{d} a, \quad\left\langle t_{\perp}^{(2)}\right\rangle_{e}=\frac{1}{\mathcal{A}} \int_{\partial C^{(2), e}} t_{\perp} \mathrm{d} a, \quad\left\langle\boldsymbol{t}_{\|}^{(I)}\right\rangle_{e}=\frac{1}{\mathcal{A}} \int_{\partial C^{(I), e}} \boldsymbol{t}_{\|} \mathrm{d} a,
$$

where $(\bullet)_{e}$ labels the exterior surface for clarity, help conveniently deliver the compact result

$$
\frac{1}{\mathcal{A}} \int_{\partial C} \boldsymbol{t} \cdot \boldsymbol{v} \mathrm{d} a=-\left\{\bar{t}_{N}\left\langle v_{\perp}^{(1)}\right\rangle_{e}-\left\langle t_{\perp}^{(2)}\right\rangle_{e} \bar{v}_{N}\right\}+\left\{\left\langle\boldsymbol{t}_{\|}^{(1)}\right\rangle_{e} \cdot \overline{\boldsymbol{v}}_{T}^{(1)}+\left\langle\boldsymbol{t}_{\|}^{(2)}\right\rangle_{e} \cdot \overline{\boldsymbol{v}}_{T}^{(2)}\right\}
$$

\subsubsection{Mechanical scale transition}

Substitution of the expressions obtained above into (3.1) delivers, after rearrangement,

$$
\left\{\left\langle\boldsymbol{t}_{\|}^{(1)}\right\rangle_{e} \cdot \overline{\boldsymbol{v}}_{T}^{(1)}+\left\langle\boldsymbol{t}_{\|}^{(2)}\right\rangle_{e} \cdot \overline{\boldsymbol{v}}_{T}^{(2)}\right\}=\left\{\bar{t}_{N}\left\langle v_{\perp}^{(1)}\right\rangle_{e}-\left\langle t_{\perp}^{(2)}\right\rangle_{e} \bar{v}_{N}\right\}+\langle\mathcal{F}\rangle+\left\langle\mathcal{V}^{(1)}\right\rangle+\left\langle\mathcal{V}^{(2)}\right\rangle+\frac{1}{\mathcal{A}} \int_{C_{o}}\left\{\dot{\psi}+\eta \dot{\theta}+\mathcal{D}+\rho_{o}(\ddot{\boldsymbol{x}}-\boldsymbol{b}) \cdot \boldsymbol{v}\right\} \mathrm{d} V .
$$


Terms appearing in this equality are expected to be time-oscillatory due to the generally dynamic nature of sliding contact but also due to the continuously varying contact interface even if the computational procedure employs a quasistatic setting. In particular, the micromechanical testing procedure induces a pointwise cyclic loading of the material in the vicinity of the contact interface. Therefore, each term is expected to oscillate about a mean once a limit cycle is reached for the test, which is characterized by the irrecoverable (damage) internal variables saturating to constant values and the recoverable (viscous) internal variables moving through cyclic variations.

To clarify the limit cycle behavior further, time-averaging

$$
(\cdot)^{\mathbf{v}}=\frac{1}{T_{\mathrm{avg}}} \int_{t_{0}}^{t_{0}+T_{\mathrm{avg}}}(\cdot) \mathrm{d} t
$$

is applied to (3.12) where $t_{o}$ is the starting time, chosen such that the initially strong transients during compression and at the start of macroscopic sliding are excluded, and $T_{\text {avg }}$ is the duration of averaging, suitably chosen in order to ensure a convergent moving time average. Recalling the time-independent BCs (Section 3.1.2), this delivers

$$
\left\langle\boldsymbol{t}_{\|}^{(1)}\right\rangle_{e}^{\mathbf{v}} \cdot \overline{\boldsymbol{v}}_{T}^{(1)}+\left\langle\boldsymbol{t}_{\|}^{(2)}\right\rangle_{e}^{\mathbf{v}} \cdot \overline{\boldsymbol{v}}_{T}^{(2)}=\bar{t}_{N}\left\langle v_{\perp}^{(1)}\right\rangle_{e}^{\boldsymbol{\nabla}}-\left\langle t_{\perp}^{(2)}\right\rangle_{e}^{\mathbf{\nabla}} \bar{v}_{N}+\langle\mathcal{F}\rangle^{\boldsymbol{\nabla}}+\left\langle\mathcal{V}^{(1)}\right\rangle^{\boldsymbol{\nabla}}+\left\langle\mathcal{V}^{(2)}\right\rangle^{\boldsymbol{\nabla}}+\frac{1}{\mathcal{A}} \int_{C_{o}}\left\{\dot{\psi}+\eta \dot{\theta}+\mathcal{D}+\rho_{o}(\ddot{\boldsymbol{x}}-\boldsymbol{b}) \cdot \boldsymbol{v}\right\}^{\boldsymbol{\nabla}} \mathrm{d} V,
$$

effectively eliminating the rapid oscillations in time. A series of observations then constitutes a basis for the mechanical scale transition:

- Due to the physical length scales involved in the micromechanical analysis, the body force contribution is negligible in magnitude.

- The sample does not experience a net change in the mean kinetic energy, hence the term involving the acceleration drops out.

- $\mathcal{D}$ vanishes over time since the damage variables saturate to constant values.

- The mean Helmholtz energy does not change. Likewise, the entropic contributions time-average to zero - see Section 5.1.3.

- The bulk dissipation occurs only in the vicinity of the contact interface where cyclic loading is experienced by the material. Therefore the integral of $\mathcal{V}$, hence its surface average, is bounded even if the sample has an arbitrarily large height.

- Based on the notation of Section 2.2, $\overline{\boldsymbol{t}}_{T}=-\left\langle\boldsymbol{t}_{\|}^{(1)}\right\rangle_{e}^{\mathbf{V}}$ represents the macroscopic tangential traction. Due to vanishing mean kinetic energy change, $\left\langle\boldsymbol{t}_{\|}^{(2)}\right\rangle_{e}^{\nabla}=\overline{\boldsymbol{t}}_{T}$ is implied. The accompanying macroscopic slip velocity is $\overline{\boldsymbol{v}}_{T}=\overline{\boldsymbol{v}}_{T}^{(1)}-\overline{\boldsymbol{v}}_{T}^{(2)}$. ( $\overline{\boldsymbol{v}}_{T}^{(2)}=\mathbf{0}$ will be employed.)

- The sample is maintained at a fixed macroscopic pressure $\bar{t}_{N}$. Hence, there is no continuous compression so that $\left\langle v_{\perp}^{(1)}\right\rangle_{e}^{\nabla}=\bar{v}_{N}$ and $\left\langle t_{\perp}^{(2)}\right\rangle_{e}^{\nabla}=\bar{t}_{N}$ are implied. $\left(\bar{v}_{N}=0\right.$ will be employed. $)$

In summary, the final form of (3.14) is

$$
\overline{\mathcal{F}}=-\overline{\boldsymbol{t}}_{T} \cdot \overline{\boldsymbol{v}}_{T}=\langle\mathcal{F}\rangle^{\boldsymbol{V}}+\left\langle\mathcal{V}^{(1)}\right\rangle+\left\langle\mathcal{V}^{(2)}\right\rangle \geq 0
$$

which represents a micro-macro mechanical dissipation equality since

1. $\overline{\mathcal{F}}$ is the total macroscopic mechanical contact interface dissipation, presently modeled as a pure surface effect associated with macroscopic frictional slip, while

2. the averaged terms represent the total microscopic dissipation that is composed of (i) a surface dissipation contribution associated with frictional slip and (ii) bulk dissipation contributions from the contacting boundary layers due to recoverable (viscous) internal variables.

$\overline{\mathcal{F}}$ is strictly non-negative due to thermodynamically consistent microscale surface and bulk formulations employed (Section $5)$, thereby satisfying the requirement of a thermodynamically consistent macroscopic contact formulation.

In view of the result (3.15), a macroscopic frictional response is observed even in the absence of microscopic friction $(\mathcal{F}=0)$ if either of the bodies are endowed with recoverable internal variables. Such a response will be of a non-Coulomb type due to the rate-dependent nature of the bulk dissipation. On the other hand, even in the absence of microscopic recoverable inelasticity $\left(\mathcal{V}^{(I)}=0\right)$ the macroscopic frictional response may be qualitatively significantly different from the microscopic one. For instance, due to the deformation of the rough contact interface $\partial C^{r}$, a non-Amontons type macroscopic response can be observed even if the microscopic model employs a constant coefficient of friction. In general, a nonAmontons and non-Coulomb type anisotropic response is expected. The constitutive formulation of this macroscopic response will not be pursued in this work - see Jones and Papadopoulos (2006) and Konyukhov et al. (2008) for aspects of anisotropic friction, and Laursen (2003) and Ronsin and Coeyrehourcq (2001) for the rate- and state-dependent frictional response of elastomers.

The micromechanical discussion of the mechanical energy balance in this section constitutes an extension of the well-known results for the contact of rubber friction on rough rigid surfaces to the case where both bodies in contact are deformable. Moreover, this approach constitutes a basis for the discussion of the thermal energy balance, which is carried out next. 


\subsection{Thermal contact homogenization}

For the space-time homogenization of the thermal contact interactions, the energy balance (2.4) is considered in integral form separately for each body:

$$
\int_{C_{0}^{(I)}} c \dot{\theta} \mathrm{d} V=\int_{C_{0}^{(I)}}(\mathcal{V}+\mathcal{D}-\mathcal{H}) \mathrm{d} V+\int_{\partial C^{(I)}} h \mathrm{~d} a+\int_{C_{o}^{(I)}} \rho_{o} r \mathrm{~d} V
$$

In this case, the bulk integral requires no further simplification although a detailed treatment of the heating term $\mathcal{H}$ is needed. This will be carried out in Section 5.1.3.

\subsubsection{Surface integrals}

The normal heat flux contribution to the right-hand side of (3.16) is

$$
\int_{\partial C^{(I)}} h \mathrm{~d} a=\int_{\partial C^{(I), i}} h \mathrm{~d} a+\int_{\partial C^{(I), r}} h \mathrm{~d} a+\int_{\partial C^{(I), e}} h \mathrm{~d} a
$$

where the integral over the inner surfaces again vanishes due to periodic BCs. For a compact representation, the following definitions will be employed:

$$
\left\langle h^{c}\right\rangle=\frac{1}{\mathcal{A}} \int_{\Gamma^{c}} h \mathrm{~d} a, \quad\left\langle\gamma^{(I)} \mathcal{F}\right\rangle=\frac{1}{\mathcal{A}} \int_{\Gamma^{c}} \gamma^{(I)} \mathcal{F} \mathrm{d} a .
$$

Upon substituting the flux representations (2.11) into the integral in (3.17) on the rough interface, which is non-zero only on the contact interface $\Gamma^{c}$, one obtains

$$
\frac{1}{\mathcal{A}} \int_{\partial C^{(I), r}} h \mathrm{~d} a=\frac{1}{\mathcal{A}} \int_{\Gamma^{c}} h^{(I)} \mathrm{d} a=\left\langle h^{(I)}\right\rangle=\left\{\begin{array}{l}
I=1:\left\langle h^{c}\right\rangle+\left\langle\gamma^{(1)} \mathcal{F}\right\rangle \\
I=2:-\left\langle h^{c}\right\rangle+\left\langle\gamma^{(2)} \mathcal{F}\right\rangle
\end{array}\right.
$$

which is accompanied by, as a consequence of $(2.7)_{2}$ or (2.12),

$$
\left\langle h^{(1)}\right\rangle+\left\langle h^{(2)}\right\rangle=\langle\mathcal{F}\rangle .
$$

Finally, the last integral in (3.17) over the exterior observation surfaces is associated with

$$
\left\langle h^{(I)}\right\rangle_{e}=\frac{1}{\mathcal{A}} \int_{\partial C^{(I), e}} h \mathrm{~d} a .
$$

\subsubsection{Thermal scale transition}

Rearrangement of (3.16) delivers

$$
-\int_{\partial C^{(I), e}} h \mathrm{~d} a=\int_{\partial C^{(I), r}} h \mathrm{~d} a+\int_{C_{o}^{(I)}} \mathcal{V} \mathrm{d} V+\int_{C_{o}^{(I)}}\left\{\mathcal{D}-\mathcal{H}+\rho_{o}(r-c \dot{\theta})\right\} \mathrm{d} V .
$$

After scaling by $\mathcal{A}$ and applying time-averaging (3.13) one obtains

$$
-\left\langle h^{(I)}\right\rangle_{e}^{\nabla}=\left\langle h^{(I)}\right\rangle^{\nabla}+\left\langle\mathcal{V}^{(I)}\right\rangle^{\boldsymbol{\nabla}}+\frac{1}{\mathcal{A}} \int_{C_{o}^{(I)}}\left\{\mathcal{D}-\mathcal{H}+\rho_{o}(r-c \dot{\theta})\right\}^{\boldsymbol{\nabla}} \mathrm{d} V .
$$

Thermal scale transition is realized via a series of observations. Those which are distinct from the mechanical case are highlighted below:

- The heat supply contribution is negligible and the mean energy change in the composite sample associated with the heat capacity term time-averages to zero.

- The heating term contribution $\mathcal{H}$ is known to be non-dissipative and hence time-averages to zero - see Section 5.1.3.

- Based on the notation of Section 2.2, the limit $\bar{h}^{(I)}=-\left\langle h^{(I)}\right\rangle_{e}^{\mathbf{V}}$ represents the macroscopic normal heat flux associated with the contacting surfaces.

- The contact contribution $\left\langle h^{c}\right\rangle$ saturates to a limit that represents the macroscopic thermal contact interactions due to conduction.

Consequently, the final form of (3.22) for each body is the macroscopic counterpart of (2.11):

$$
\bar{h}^{(1)}=\left\langle h^{c}\right\rangle^{\mathbf{V}}+\left\langle\gamma^{(1)} \mathcal{F}\right\rangle^{\mathbf{V}}+\left\langle\mathcal{V}^{(1)}\right\rangle^{\boldsymbol{\nabla}}, \quad \bar{h}^{(2)}=-\left\langle h^{c}\right\rangle+\left\langle\gamma^{(2)} \mathcal{F}\right\rangle^{\mathbf{\nabla}}+\left\langle\mathcal{V}^{(2)}\right\rangle^{\mathbf{v}}
$$

which is a micro-macro thermal dissipation equality since

1. $\bar{h}^{(I)}$ on the left-hand sides control the entropy flux through the macroscopic contact interface and hence are associated 
with the macroscopic thermal contact interface dissipation, while

2. the right-hand sides in (3.24) are associated with surface dissipation due to contact conduction and friction as well as heating due to bulk dissipation.

Together with its mechanical counterpart (3.15), (3.24) ensures that dissipation is preserved through the scale transition. Hence, they contribute to the thermodynamic consistency of the homogenization framework, which will be referred to as multiscale thermodynamic consistency. Moreover, the sum of $\bar{h}^{(I)}$ delivers, upon making use of (2.12) and (3.15),

$$
\bar{h}^{(1)}+\bar{h}^{(2)}=\overline{\mathcal{F}}
$$

thereby establishing another aspect of multiscale thermodynamic consistency since this result is the direct macroscopic counterpart of $(2.7)_{2}$. If there is no microscopic mechanical dissipation or if stick conditions prevail, one obtains $\bar{h}^{(1)}=-\bar{h}^{(2)}$.

\subsubsection{Macroscopic dissipation partitioning}

Introducing $\bar{h}^{c}=\left\langle h^{c}\right\rangle^{\nabla}$, the result (3.24) may be stated in a form that is similar to (2.11) to highlight the conductive and frictional parts of the macroscopic contact heat flux via

$$
\bar{h}^{(1)}=\bar{h}^{c}+\bar{\gamma}^{(1)} \overline{\mathcal{F}}, \quad \bar{h}^{(2)}=-\bar{h}^{c}+\bar{\gamma}^{(2)} \overline{\mathcal{F}}
$$

if one defines the macroscopic relative effusivities $\bar{\gamma}^{(I)}$

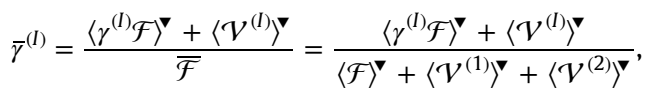

which satisfy $\bar{\gamma}^{(1)}+\bar{\gamma}^{(2)}=1$ by construction. As a consequence, one observes the following:

1. If there are no microscopic recoverable internal variables then $\bar{\gamma}^{(I)}$ are associated only with the frictional dissipation partitioning on the microscale: $\bar{\gamma}^{(I)}=\left\langle\gamma^{(I)} \mathcal{F}\right\rangle^{\nabla} /\langle\mathcal{F}\rangle^{\boldsymbol{V}}$. However, since the contact pressure and frictional tractions are highly non-uniform, $\gamma^{(I)}$ spatially vary over $\Gamma^{c}$, i.e. $\bar{\gamma}^{(I)} \neq \gamma^{(I)}$ in general. Only if $\gamma^{(I)}$ are constants does one obtain $\bar{\gamma}^{(I)}=\gamma^{(I)}$.

2. If there is no microscopic friction then macroscopic frictional dissipation is due to $v$ only. In this case, one obtains a non-

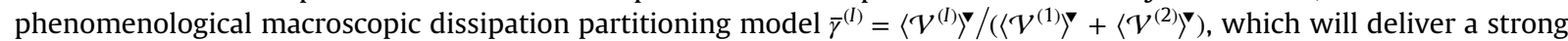
dependence on the macroscopic contact variables due to the similar dependence of $\mathcal{V}$. Clearly, if only one of the bodies is dissipative then the macroscopic frictional dissipation is momentarily transmitted entirely to the contact surface that is associated with this dissipating body, for instance in the case of rubber sliding on a hard surface. Ensuing thermal interaction will retransfer heat to the non-dissipative body via macroscopic contact conduction $\left(\bar{h}^{c}\right)$.

The expression (3.27) completes the multiscale framework for the determination of not only the frictional dissipation but also its partitioning onto the macroscopic surfaces in contact. Moreover, since the microscale contribution $\mathcal{F}$ can also be based on lower-scale dissipation mechanisms that would similarly deliver $\gamma^{(I)}$, the overall framework is able to predict how dissipation accumulates and is partitioned across multiple scale transitions.

\section{Algorithmically consistent homogenization framework}

\subsection{Macroscopic temperature jump}

When there is no macroscopic slip $\left(\overline{\boldsymbol{v}}_{T}=\boldsymbol{0}\right)$, the multiscale contact problem condenses to finding the relation between the conductive heat flux $\bar{h}^{c}$ and the macroscopic temperature jump $\bar{\vartheta}_{c}=\bar{\theta}^{(1)}-\bar{\theta}^{(2)}$ for two deformable boundary layers with rough surfaces at a macroscopic contact pressure $\bar{t}_{N}$. It is convenient to cast the problem in a setting where the master surface temperature $\bar{\theta}^{(2)}$ and $\bar{h}^{c}$ are prescribed and $\bar{\vartheta}_{c}$ is sought. A contact homogenization approach that is suitable for this purpose at the finite deformation regime with finite temperature jumps has been proposed in Temizer (2014a) and is

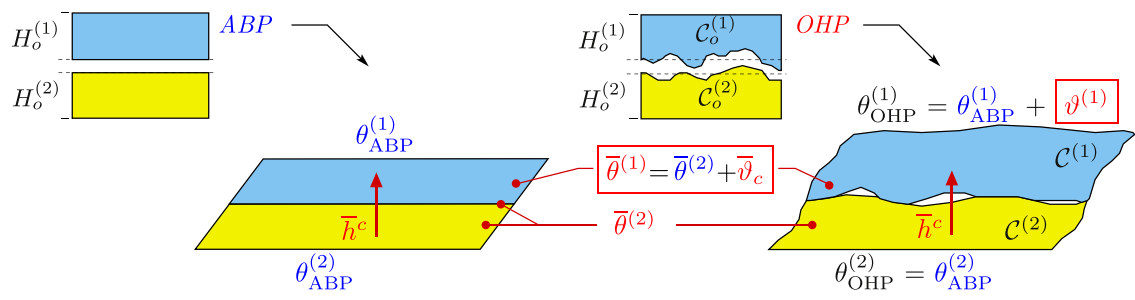

Fig. 2. The accompanying bar problem (ABP) and the original heterogeneous problem (OHP) with the explicit enforcement of the macroscopic contact surface temperatures $\bar{\theta}^{(I)}$. When there is no macroscopic slip, the procedure only presses the samples against each other at $\bar{t}_{N}$. 
incorporated into the present framework as summarized in Fig. 2 - see Temizer (2014a) for extensive references. The algorithmic stages in a computational framework are:

1. Accompanying Bar Problem $(A B P)$ : The ABP represents a homogeneous microscale contact problem where the master surface is at a uniform temperature $\bar{\theta}^{(2)}$. By enforcing this condition at the flat contact interface and imposing $\bar{h}^{c}$, the ABP is solved to obtain $\theta_{\mathrm{ABP}}^{(I)}$.

2. Original Heterogeneous Problem $(O H P)$ : The macroscopic master surface temperature is indirectly projected into the original heterogeneous sample by enforcing $\theta_{\mathrm{OHP}}^{(2)}=\theta_{\mathrm{ABP}}^{(2)}$ on $\partial C^{(2), e}$ while $\bar{h}^{c}$ is imposed on $\partial C^{(1), e}$ (see Fig. 1) which delivers the difference $\vartheta^{(1)}=\theta_{\mathrm{OHP}}^{(1)}-\theta_{\mathrm{ABP}}^{(1)}$

Subsequent to the solution of the OHP, the macroscopic temperature jump is extracted as follows:

- For insufficiently large sample heights $H_{o}^{(I)}, \theta_{\mathrm{OHP}}^{(1)}$ and, therefore, $\vartheta^{(1)}$ are oscillatory on $\partial C^{(1), e}$. As $H_{o}^{(I)}$ increase, $\partial C^{(1), e}$ becomes flat and $\vartheta^{(1)}$ a constant. Nevertheless, it is convenient to define a surface average for arbitrarily sized samples:

$$
\left\langle\vartheta^{(1)}\right\rangle_{e}=\frac{1}{\mathcal{A}} \int_{\partial \mathcal{C}^{(1), e}} \vartheta^{(1)} \mathrm{d} a
$$

- The framework will remain identical when there is macroscopic slip as well. In this case, however, even when all microscopic dissipation is omitted, the microstructural contact interface topography can change significantly as the slave sample slides over the master, leading to a $\vartheta^{(1)}$ that rapidly oscillates in time. One identifies $\bar{\vartheta}_{c}=\left\langle\vartheta^{(1)}\right\rangle_{e}^{\nabla}$ as the macroscopic jump.

\subsection{Explicit enforcement of contact surface temperatures}

The thermodynamic consistency of the contact homogenization framework is ensured by the constructions of Sections 3.2 and 3.3. However, if a fully coupled thermomechanical analysis framework is pursued in Section 4.1, the choice of macroscopic slave/master can influence the value of $\bar{\vartheta}_{c}$ and, for a given choice, $\bar{\vartheta}_{c}$ does not saturate to a limit, contrary to expectation (Temizer, 2014a). Moreover, in the case with macroscopic slip, the continuous heating of the samples by microscopic frictional and viscous dissipation can have an arbitrarily large influence on the temperature-dependent material response such that the moving time averages may not saturate to a limit with increasing averaging time. With the aim of restoring algorithmic consistency so that purely numerical choices do not have an influence on the homogenization outcome, an explicit enforcement of the macroscopic contact surface temperatures $\bar{\theta}^{(I)}$ will be pursued following Temizer (2014a). A similar condition has been enforced for the viscosity in Reinelt (2009) in order to obtain a convergent macroscopic friction response. Specifically, it is proposed that all temperature-dependent contact and material model parameters be explicitly evaluated at $\bar{\theta}^{(1)}$ for the slave and at $\bar{\theta}^{(2)}$ for the master. Such an approach induces an incrementally two-phase micromechanical test with optimal computational effort where an exact staggered solution scheme is naturally applicable at any time step:

1. Mechanical phase: A purely mechanical nonlinear contact problem is solved. The mechanical response remains temperature-dependent although the effect of the temperature is not via its local value induced by $\bar{h}^{c}$ throughout a sample but rather explicitly through $\bar{\theta}^{(I)}$. As an example for future reference, the viscous dissipation in the slave is $\mathcal{V}=\boldsymbol{\tau}_{v}^{\mathrm{dev}} \cdot \boldsymbol{\tau}_{v}^{\mathrm{dev}} / 2 \eta\left(\bar{\theta}^{(1)}\right)$ where $\tau_{v}^{\mathrm{dev}}=f_{v}\left(\bar{\theta}^{(1)}\right) G_{v} \mu_{o v} \mathcal{T}_{v}^{\mathrm{dev}}\left(\boldsymbol{b}_{e}^{\text {iso }}\right)-$ see Appendix A.

2. Thermal phase: A purely thermal linear contact problem is solved within which all deformation is frozen including the contact pressure and any further changes to the contact active set (Laursen, 2003; Wriggers, 2006). The deformationdependent thermal response is preserved without modification but temperature-dependent thermal properties are evaluated at $\bar{\theta}^{(I)}$. For instance, the thermal conductivity in the slave is $k=k_{0}+k_{1}\left(\bar{\theta}^{(1)}-\theta_{0}\right)-$ see Section 5.1.

The motivation for this approach is closely related to the classical separation of scales assumption for homogenization in thermomechanics of heterogeneous media (Yu and Fish, 2002; Temizer and Wriggers, 2011). As the ratio $\varepsilon$ of a representative microscopic length scale to a macroscopic one becomes smaller, the surfaces essentially become smoother such that $\bar{\vartheta}_{c}$ goes to zero. Close to this limit, the temperature within the boundary layer in the vicinity of the contact interface is uniform at the master surface temperature $\bar{\theta}^{(2)}$, i.e. $\bar{\theta}^{(1)}=\bar{\theta}^{(2)}$, and the procedure described above becomes exact. However, an important difference for the class of problems investigated is that the absolute value of $\varepsilon$ is important since the ability to characterize $\bar{\theta}_{c}$ of finite magnitude is of concern. Moreover, different $\varepsilon$ induce viscous dissipation at different loading frequencies such that the macroscopic frictional response, and hence the frictional contribution to the macroscopic heat flux, will vary. For $\varepsilon$ that is sufficiently small for homogenization to be of practical importance but not vanishingly small, the two-phase approach is approximate. In Temizer (2014a), it has been shown it can make accurate predictions of $\bar{\vartheta}_{c}$. Moreover, the projection of the macroscopic slave contact surface temperature $\bar{\theta}^{(1)}=\bar{\theta}^{(2)}+\bar{\vartheta}_{c}$ into the slave sample rather than $\bar{\theta}^{(2)}$ can yield a slightly better representation of the original heterogeneous response, and was therefore referred to as a first-order corrector to the initial scheme motivated by scale separation. The first-order correction leads to a higher computational cost because it leads to an iterative homogenization scheme in view of the fact that the value of $\bar{\theta}^{(1)}$ is based 
on the homogenization output $\bar{\vartheta}_{c}$. It will be omitted in the numerical investigations of this work.

\subsection{Overall homogenization framework}

In order to carry out the solution of a macroscopic sliding contact problem in a standard numerical setting, the microscopic problem must deliver $\left\{\overline{\boldsymbol{t}}_{T}, \bar{h}^{c}, \bar{\gamma}^{(1)}, \bar{\gamma}^{(2)}\right\}$ for given $\left\{\bar{t}_{N}, \overline{\boldsymbol{v}}_{T}, \bar{\theta}^{(2)}, \bar{\vartheta}_{c}\right\}$, with $\bar{\theta}^{(1)}=\bar{\theta}^{(2)}+\bar{\vartheta}_{c}$. Hence, there are three objectives: (a) the determination of $\overline{\boldsymbol{t}}_{T}$ through $\overline{\mathcal{F}}$ based on (3.15), (b) the determination of the partitioning of $\overline{\mathcal{F}}$ through $\bar{\gamma}^{(I)}$ based on (3.27), and finally (c) the determination of the relation between $\bar{h}^{c}$ and $\bar{\vartheta}_{c}$. The procedure described in the earlier section was motivated by the third objective but is applicable to all three objectives and also has the advantage that it decouples the first two from the last one. For objectives (a) and (b), one can directly employ the inputs $\left\{\bar{t}_{N}, \overline{\boldsymbol{v}}_{T}, \bar{\theta}^{(2)}, \bar{\vartheta}_{c}\right\}$ in a purely mechanical test where the samples are kept at their respective temperatures - the solution of a thermal problem is not required. The realization of objective (c) encompasses (a) and (b) by construction, although at a comparatively higher computational cost. In (c), the natural input is $\left\{\bar{t}_{N}, \overline{\boldsymbol{v}}_{T}, \bar{\theta}^{(2)}, \bar{h}^{c}\right\}$, delivering $\left\{\overline{\boldsymbol{t}}_{T}, \bar{\vartheta}_{c}, \bar{\gamma}^{(1)}, \bar{\gamma}^{(2)}\right\}$. But the procedure is implicit since $\bar{\theta}^{(1)}$ depends on $\bar{\vartheta}_{c}$, which is not known beforehand when $\bar{h}^{c}$ is the input. Nevertheless, self-consistency iterations starting from the initial guess $\bar{\vartheta}_{c}=0$ were observed to converge rapidly and once the relation of $\bar{\vartheta}_{c}$ to $\bar{h}^{c}$ is determined it may be inverted for use with $\bar{\vartheta}_{c}$ as the input. This relation will be different from the predictions of standard micromechanical models for thermal contact (Madhusudana, 1996; Bahrami et al., 2006), where only compressive loading is considered, mainly because the contact interface topography presently also depends on the macroscopic slip velocity due to the rate-dependent material response. Consequently, the overall framework is additionally able to evaluate the macroscopic coupling between sliding and conductive contact heat flux (Chantrenne and Raynaud, 1997; Laraqi, 1997; Salti and Laraqi, 1999). To summarize, a single purely mechanical test is sufficient (i) if only macroscopic frictional dissipation and its partitioning onto the contacting surfaces are of concern, or (ii) if $\bar{\vartheta}_{c} \approx 0$ such that the macroscopic solution scheme can directly enforce $\bar{\vartheta}_{c}=0$. On the other hand, if $\bar{\vartheta}_{c}$ is not negligible and the modeling of $\bar{h}^{c}$ is required, then one can directly pursue the incrementally twophase micromechanical test, complemented by self-consistency iterations. The algorithmic flow of the micromechanical test is outlined in Fig. 3.

\section{Material and contact modeling}

The modeling approach is briefly summarized in order to provide a basis for the numerical investigations as well as to clarify various observations stated earlier without proof.

\subsection{Isotropic thermo-viscoelasticity with damage}

The heat flux model follows the classical Fourier's law $\boldsymbol{q}=-\mathbf{k g}$ with $\mathbf{g}$ being the spatial temperature gradient, where the conductivity $k$ is in general temperature-dependent via constants $k_{o}$ and $k_{1}: k=k_{o}+k_{1} \vartheta$. The remaining constitutive behavior is motivated by rubber-like materials and follows the finite thermo-viscoelasticity model developed in Reese and Govindjee (1998a), which is based on the viscoelasticity approach of Reese and Govindjee (1998b) combined with the thermoelasticity framework of Chadwick (1974) and Chadwick and Creasy (1984). As straightforward extensions, Mullins-

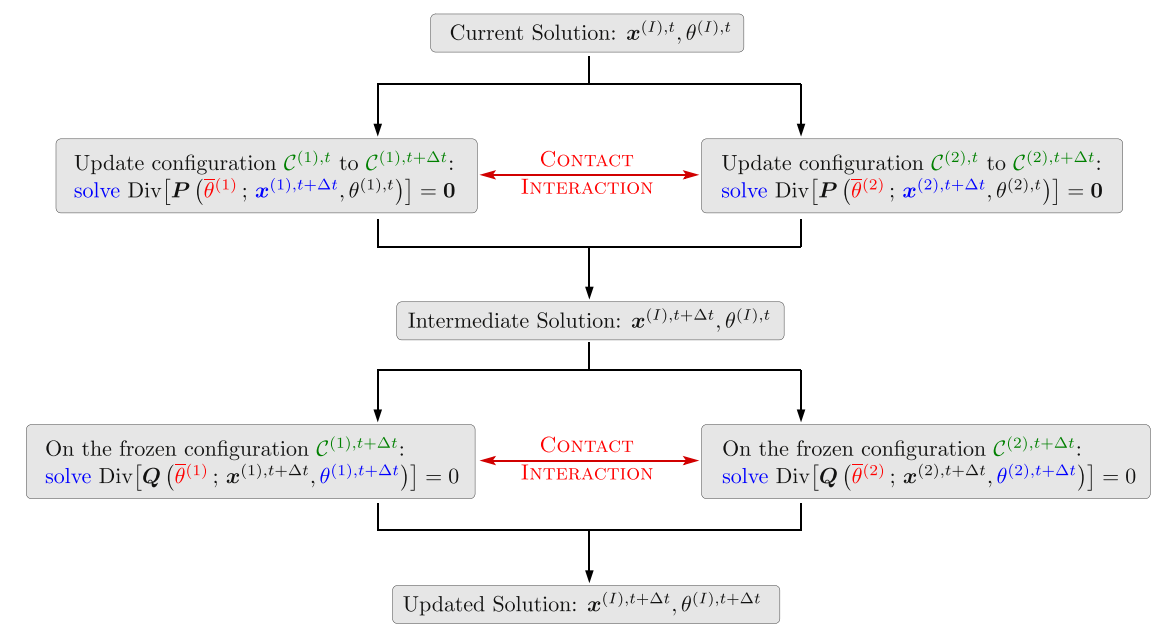

Fig. 3. Algorithmic flow for a single time step of ABP and OHP (Fig. 2) is outlined together with the microscopic balance invoked at the mechanical and thermal phases of the micromechanical test. The microscopic stress $\boldsymbol{P}$ and the heat flux $\boldsymbol{Q}$ within each $C^{(I)}$ depend on a parameter set $\{\bar{\theta} ; \boldsymbol{x}, \theta\}$. All temperature dependent quantities are explicitly evaluated at $\bar{\theta}$ (Section 4.2) and, hence, are not influenced by the temperature distribution $\theta$ obtained within the thermal phase. 
type damage is incorporated and the temperature dependence of the viscosity is taken into account. Specific functional choices for the material behavior that are not explicitly stated below are summarized in Appendix A.

\subsubsection{Framework fundamentals}

The isotropic material model is an extension of the standard linear solid (SLS) to the finite deformation regime. Introducing the internal variable set $I=\left\{\boldsymbol{C}_{v}^{-1}, G_{e}, G_{v}\right\}$, the SLS-type split

$$
\psi=\psi_{e}\left(\boldsymbol{b}, \theta, G_{e}\right)+\psi_{v}\left(\boldsymbol{b}_{e}, \theta, G_{v}\right)+\int_{\theta_{o}}^{\theta}\left(1-\frac{\theta}{\theta^{\prime}}\right) c_{o} \mathrm{~d} \theta^{\prime}
$$

is admitted where the constant heat capacity $c_{o}$ from linear thermoelasticity has been condensed out. The split is based on the idea that $\boldsymbol{F}=\boldsymbol{F}_{e} \boldsymbol{F}_{v}$ and $\boldsymbol{F}_{e}$ control, respectively, the stress contributions from the elastic and viscous (Maxwell) branches through $\boldsymbol{b}=\boldsymbol{F F}^{T}$ and $\boldsymbol{b}_{e}=\boldsymbol{F C}_{v}^{-1} \boldsymbol{F}^{T}$ with $\boldsymbol{C}_{v}=\boldsymbol{F}_{v}^{T} \boldsymbol{F}_{v}$. Additionally, the moduli of each branch can degrade through a factor $G_{x}$ $(x=e$ or $v$ ) with 1 corresponding to the undamaged material and 0 representing full damage. A general, purely mechanical response choice in (2.5) that is consistent with this framework is

$$
\psi_{o}=\psi_{o e}\left(\boldsymbol{b}, G_{e}\right)+\psi_{o v}\left(\boldsymbol{b}_{e}, G_{v}\right), \quad e_{o}=e_{o e}\left(\boldsymbol{b}, G_{e}\right)+e_{o v}\left(\boldsymbol{b}_{e}, G_{v}\right) .
$$

Under mild assumptions (Reese and Govindjee, 1998a) on the last contribution to $\psi$ in (2.5), the final form of (5.1) is

$$
f_{x}(\theta)=\frac{\theta}{\theta_{0}}+g_{x}(\theta)-g_{x}\left(\theta_{0}\right)-\left.\vartheta \frac{\partial g_{x}}{\partial \theta}\right|_{\theta=\theta_{0}} \longrightarrow \psi_{x}=f_{x} \psi_{o x}-\frac{\vartheta}{\theta_{o}} e_{o x},
$$

essentially reflecting the nonlinear temperature dependence of the moduli and also implying

$$
c=c_{o}-\theta \frac{\partial^{2} g_{e}}{\partial \theta^{2}}-\theta \frac{\partial^{2} g_{v}}{\partial \theta^{2}} .
$$

\subsubsection{Modeling details}

Modeling details are based on linear thermoelasticity parameters $\kappa_{o x}$ (bulk modulus), $\mu_{o x}$ (shear modulus) and $\alpha_{o x}$ (linear thermal expansion coefficient). $e_{o x}$ contribute to thermal expansion via

$$
e_{o e}=3 \kappa_{o e} \alpha_{o e} \theta_{o} \mathcal{E}_{e}(\operatorname{det} \boldsymbol{b}), \quad e_{o v}=3 \kappa_{o v} \alpha_{o v} \theta_{o} \mathcal{E}_{v}\left(\operatorname{det} \boldsymbol{b}_{e}\right)
$$

The predominant mechanical response is delivered through the volumetric-deviatoric decoupling

$$
\begin{aligned}
& \psi_{o e}=\psi_{o e}^{\mathrm{vol}}+\psi_{o e}^{\mathrm{dev}}=\kappa_{o e} \Psi_{e}^{\mathrm{vol}}(\operatorname{det} \boldsymbol{b})+G_{e} \mu_{o e} \Psi_{e}^{\mathrm{dev}}\left(\boldsymbol{b}^{\mathrm{iso}}\right) \\
& \psi_{o v}=\psi_{o v}^{\mathrm{vol}}+\psi_{o v}^{\mathrm{dev}}=\kappa_{o v} \Psi_{v}^{\mathrm{vol}}\left(\operatorname{det} \boldsymbol{b}_{e}\right)+G_{v} \mu_{o v} \Psi_{v}^{\mathrm{dev}}\left(\boldsymbol{b}_{e}^{\mathrm{iso}}\right)
\end{aligned}
$$

of the strain energy functions where the definition $\boldsymbol{A}^{\text {iso }}=(\operatorname{det} \boldsymbol{A})^{-1 / 3} \boldsymbol{A}$ represents the isochoric part of a tensor $\boldsymbol{A}$. Above, damage has been omitted from all volumetric contributions.

In summary, using the parameter-normalized functions $\Psi_{x}^{\mathrm{vol} / \mathrm{dev}}$ and $\mathcal{E}_{x}$ in (5.3), the specific formulation (5.1) of the general Helmholtz energy representation (2.5) employs

$$
\psi_{x}=f_{x}\left(\kappa_{o x} \Psi_{x}^{\mathrm{vol}}+G_{x} \mu_{o x} \Psi_{x}^{\mathrm{dev}}\right)-3 \kappa_{o x} \alpha_{o x} \vartheta \mathcal{E}_{x} .
$$

Within this model, using $\bar{\psi}_{x}^{\mathrm{dev}}$ to denote the deviatoric energy stored by the undamaged material, the thermodynamic driving forces for the damage variables are

$$
-\frac{\partial \psi}{\partial G_{x}}=-f_{x} \frac{\partial \psi_{o x}^{\mathrm{dev}}}{\partial G_{x}}=-f_{x} \mu_{o x} \Psi_{x}^{\mathrm{dev}}=-\bar{\psi}_{x}^{\mathrm{dev}}
$$

so that $G_{x}$ must be monotonically decreasing in order to satisfy $(2.2)_{3}$. On the other hand, the driving force for the viscous internal variable is based on the Kirchhoff stress decomposition

$$
\boldsymbol{\tau}=2 \frac{\partial \psi}{\partial \boldsymbol{b}} \boldsymbol{b}=2 \frac{\partial \psi_{e}}{\partial \boldsymbol{b}} \boldsymbol{b}+2 \frac{\partial \psi_{v}}{\partial \boldsymbol{b}_{e}} \boldsymbol{b}_{e}=\tau_{e}+\boldsymbol{\tau}_{v}
$$

where the stress functions $\mathcal{T}_{x}^{\mathrm{vol} / \mathrm{dev}}$ delivered by $\Psi_{x}^{\mathrm{vol} / \mathrm{dev}}$ determine the contribution $\tau_{x}$ :

$$
\boldsymbol{\tau}_{o x}=\kappa_{o x} \mathcal{T}_{x}^{\mathrm{vol}}+G_{x} \mu_{o x} \mathcal{T}_{x}^{\mathrm{dev}} \longrightarrow \boldsymbol{\tau}_{x}=f_{x} \boldsymbol{\tau}_{o x}-3 \kappa_{o x} \alpha_{o x} \vartheta \boldsymbol{I} .
$$

Similar to damage, viscous effects are omitted from the volumetric contributions. It can then be shown (Reese and Govindjee, 1998b) that the thermodynamically consistent update of $\boldsymbol{C}_{v}^{-1}$ is naturally driven by 


$$
\boldsymbol{\tau}_{v}^{\operatorname{dev}}\left(\boldsymbol{b}_{e}^{\text {iso }}, \theta, G_{v}\right)=f_{v}(\theta) G_{v} \mu_{o v} \mathcal{T}_{v}^{\operatorname{dev}}\left(\boldsymbol{b}_{e}^{\text {iso }}\right)
$$

where the evolution rate is controlled by the temperature-dependent shear viscosity $\eta$.

\subsubsection{Time-oscillatory thermodynamic quantities}

In order to verify various observations stated without proof in Section 3.3, the energy balance is examined with the specific choice (5.1) for $\psi$. Explicitly, the heating term contribution to (2.4) is

$$
\mathcal{H}=-\theta \frac{\partial^{2} \psi}{\partial \theta \partial \boldsymbol{F}} \cdot \dot{\boldsymbol{F}}-\theta \frac{\partial^{2} \psi}{\partial \theta \partial \boldsymbol{C}_{v}^{-1}} \cdot \dot{\boldsymbol{C}_{v}^{-1}}-\theta \frac{\partial^{2} \psi}{\partial \theta \partial G_{e}} \cdot \dot{G}_{e}-\theta \frac{\partial^{2} \psi}{\partial \theta \partial G_{v}} \cdot \dot{G}_{v} .
$$

The damage variables saturate with time so that their rates eventually vanish. Concentrating on the remaining terms and making use of standard relations (Simo et al., 1998), the specific form (5.1) implies

$$
\begin{aligned}
\theta \frac{\partial^{2} \psi}{\partial \theta \partial \boldsymbol{F}} \cdot \dot{\boldsymbol{F}}+\theta \frac{\partial^{2} \psi}{\partial \theta \partial \boldsymbol{C}_{v}^{-1}} \cdot \dot{\boldsymbol{C}_{v}^{-1}} & =\theta \frac{\partial^{2} \psi_{e}}{\partial \theta \partial \boldsymbol{b}} \cdot \frac{\partial \boldsymbol{b}}{\partial \boldsymbol{F}} \dot{\boldsymbol{F}}+\theta \frac{\partial^{2} \psi_{v}}{\partial \theta \partial \boldsymbol{b}_{e}} \cdot \frac{\partial \boldsymbol{b}_{e}}{\partial \boldsymbol{F}} \dot{\boldsymbol{F}}+\theta \frac{\partial^{2} \psi_{v}}{\partial \theta \partial \boldsymbol{b}_{e}} \cdot \frac{\partial \boldsymbol{b}_{e}}{\partial \boldsymbol{C}_{v}^{-1}} \dot{\boldsymbol{C}_{v}^{-1}}=\theta \frac{\partial^{2} \psi_{e}}{\partial \theta \partial \boldsymbol{b}} \cdot \dot{\boldsymbol{b}}+\theta \frac{\partial^{2} \psi_{v}}{\partial \theta \partial \boldsymbol{b}_{e}} \cdot \dot{\boldsymbol{b}}_{e} \\
& =\theta \frac{\partial \tau_{e}}{\partial \theta} \cdot \boldsymbol{d}+\theta \frac{\partial \boldsymbol{\tau}_{v}}{\partial \theta} \cdot \boldsymbol{d}_{e}
\end{aligned}
$$

where $\boldsymbol{d}$ and $\boldsymbol{d}_{e}$ are the stretching tensors associated, respectively, with $\boldsymbol{F}$ and $\boldsymbol{F}_{e}$. Overall, after damage saturation one obtains

$$
\mathcal{H}_{e}=-\theta \frac{\partial \tau_{e}}{\partial \theta} \cdot \boldsymbol{d}, \quad \mathcal{H}_{v}=-\theta \frac{\partial \tau_{v}}{\partial \theta} \cdot \boldsymbol{d} e \quad \longrightarrow \quad \mathcal{H}=\mathcal{H}_{e}+\mathcal{H}_{v} .
$$

The terms $\mathcal{H}_{x}$ correspond to the classical non-dissipative Gough-Joule effect. Hence, $\mathcal{H}$ represents structural elastic heating after damage saturation. Consequently, its time average vanishes since the material is subjected to cyclic loading within the homogenization scheme. In a similar fashion, the time average of the entropic contribution $\eta \dot{\theta}$ to the stress power (3.2) in Section 3.2

$$
\frac{\partial \psi_{x}}{\partial \theta}=\frac{\partial f_{x}}{\partial \theta} \psi_{o x}-\frac{1}{\theta_{0}} e_{o x} \longrightarrow \eta \dot{\theta}=-\left(\frac{\partial \psi_{e}}{\partial \theta}+\frac{\partial \psi_{v}}{\partial \theta}-c_{o} \ln \frac{\theta}{\theta_{0}}\right) \dot{\theta}
$$

also vanishes once $\eta$ starts oscillating about a mean value after damage saturation.

\subsection{Thermomechanical contact with friction}

While various computational frameworks exist for handling thermomechanical contact with friction (Laursen, 2003; Wriggers, 2006), a mortar-based isogeometric framework will be pursued, which combines the advantages of (i) the numerical robustness of mortar-based contact discretizations with (ii) the surface smoothness induced by isogeometric volume discretizations. For mechanical contact with friction, such a framework has been derived in Temizer (2013) from a mixed formulation and the variational approach employed has also been extended to frictionless thermomechanical contact in Temizer (2014a). The combination of these approaches towards frictional thermomechanical contact is straightforward since only the incorporation of frictional heating is required, without any major modification to the contact algorithms. Within this combined framework, it is remarked that all contact constraints (normal, tangential and thermal) will be handled with the augmented Lagrangian method (Alart and Curnier, 1991), which is known to enhance the numerical robustness.

The slip criterion is based on an Amontons-Coulomb law with a constant friction coefficient $k_{o}$, which is the only ingredient for mechanical contact modeling. For the conduction part of the heat flux (2.11), the referential form $h_{0}^{c}=\partial \mathcal{H}_{0}^{c} / \partial \vartheta_{c}$ of (2.14) is employed with

$$
\mathcal{H}_{o}^{c}=-k_{c} p_{N}^{a} \vartheta_{c}^{2} / 2
$$

which implies a pressure-dependent contact heat flux. Here, $p_{N}$ is the referential counterpart of $t_{N}$ and $\left\{k_{c}, a\right\}$ are interface parameters. When $a=1$, one obtains the spatial form $\mathcal{H}^{c}=-k_{c} t_{N} \vartheta_{c}^{2} / 2$.

\section{Numerical investigations}

In this section, representative numerical investigations will be presented in order to demonstrate the major aspects of the space-time thermomechanical contact homogenization approach. The parameters appearing in the material and contact models of Section 2 and the accompanying Appendix A are chosen according to Appendix B. The changes from these default choices will be noted explicitly. In the default setting, both bodies are assumed to be viscoelastic and a periodic roughness is assigned only on the sliding upper (slave) sample while the softer stationary lower (master) sample surface is initially 


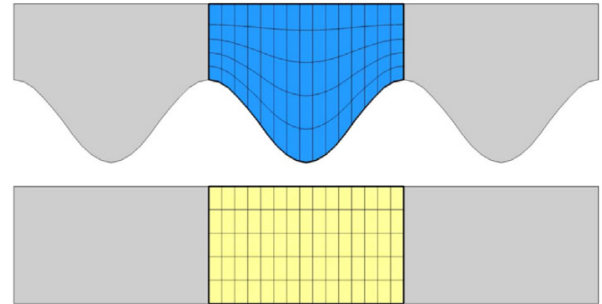

(a) unilateral roughness (default)

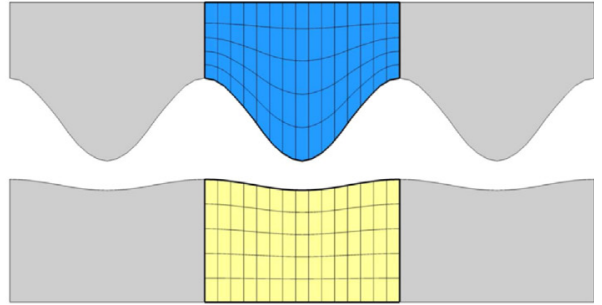

(b) bilateral roughness

Fig. 4. The periodic contact interface topographies employed are shown. The slave (blue) and master (yellow) computational cells are embedded among their periodic images. (a) Unilateral roughness (default) and (b) bilateral roughness. (For interpretation of the references to color in this figure caption, the reader is referred to the web version of this paper.)

smooth. Bilateral periodic and random roughness will be addressed in later stages. In all cases, roughness will be considered only at a single length scale. A more realistic choice from both the modeling and the physical perspectives would be the consideration of multiple scales (Ciavarella et al., 2000; Paggi and Ciavarella, 2010; Borri and Paggi, 2015).

Due to the two-dimensional simulation setting, it will be sufficient to monitor the macroscopic frictional response with a macroscopic coefficient of friction:

$$
\overline{\mathbb{k}}_{m}=\frac{\bar{t}_{T}}{\bar{t}_{N}} .
$$

$\bar{t}_{N}$ is a constant so that, when necessary, a time-averaging operation may be performed directly on an oscillatory $\overline{\mathbb{k}}_{m}$ profile to deliver a mean value. This is a mechanical definition. An alternative dissipation-based definition is

$$
\overline{\mathrm{k}}_{d}=\overline{\mathcal{F}}^{\bullet}
$$

where the normalization notation

$$
(\cdot)^{\bullet}=\frac{(\cdot)}{\bar{t}_{N} \bar{v}_{T}}
$$

has been introduced. These mechanical and dissipation-based definitions of $\bar{k}$ are expected to be equivalent in the sense of the micro-macro mechanical dissipation equality (3.15), which is to be demonstrated.

The numerical discretization of the samples is based on B-splines (Piegl and Tiller, 1996), as a specific case of isogeometric analysis with NURBS (Hughes et al., 2005). A number of algorithmic challenges associated with the imposition of large sliding contact are addressed elsewhere (Temizer, 2014b), the central one being due to the fact that the slave sample will quickly leave the span of the master sample during large sliding so that not only periodic BCs on the samples but also the periodic embedding of the samples among their images are necessary to capture ensuing contact. Periodic embedding essentially enables the incorporation of periodic boundary conditions for micromechanical contact simulations with large sliding. As in similar settings for the micromechanics of materials and interfaces, it requires geometric periodicity. For random roughness, this is ensured by modifying the portions of the original rough surfaces in the vicinity of the periodically linked inner surfaces (Fig. 1). Although this modification appears to destroy true randomness, it is localized so that with increasing sample size the statistical properties of the surfaces will be dominated by the original non-modified regions see Uchidate et al. (2011) for a discussion in the context of random roughness generation.

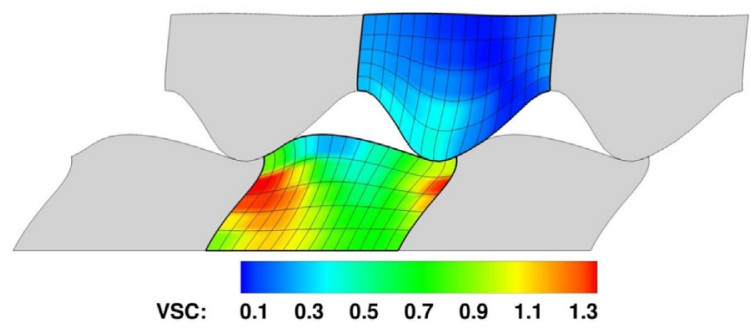

(a) $\mathrm{VSC}=\left\|\boldsymbol{C}_{v}^{-1}-\boldsymbol{I}\right\|$

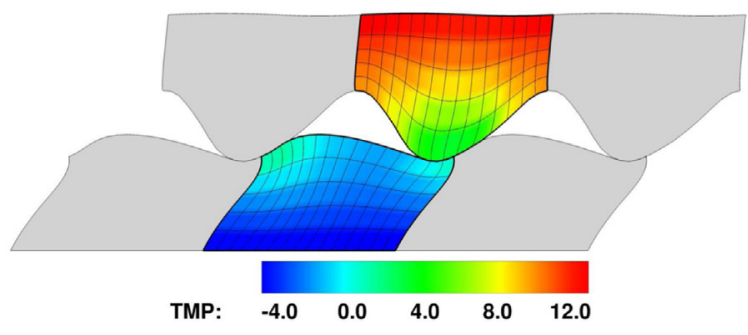

(b) $\mathrm{TMP}=\vartheta$

Fig. 5. Viscous internal variable and temperature distribution at an instance during the dragging phase for the default simulation setting. Periodic embedding ensures that the central slave and master computational cells interact not only with each other but with all of their periodic images. 


\subsection{Microscopic bulk and interface response}

Based on the simulation choices stated above, Fig. 6(a) demonstrates the effect of the microscopic material and interface response. For a flat interface, $\overline{\mathrm{k}}_{m}$ is constant at its microscopic value $\mathrm{k}_{0}$. In the case of unilateral roughness on the upper (slave) surface, a $\overline{\mathbb{k}}_{m}$ value that is larger than $\mathbb{k}_{o}$ is observed even without inelasticity effects. This deviation is strictly due to finite deformations (Temizer, 2014b; Stupkiewicz et al., 2014) and, hence, would vanish in the regime where linear elasticity is applicable for the present setup. In the default setting where the bodies are additionally viscoelastic, a significantly larger $\overline{\mathrm{k}}_{m}$ is observed due to the additional microscopic bulk dissipation and a comparable value is obtained even if the microscopic interface dissipation is neglected $\left(k_{o}=0\right)$, indicating significant viscous effects. Finally, if damage is incorporated then the deformations slightly increase due to softening but the overall effect on $\overline{\mathrm{k}}_{m}$ is not significant in this case. The slight oscillations are due to a non-uniform damage distribution across the length of the master. Damage effects will be omitted from remaining investigations.

The mechanical and dissipation-based definitions of $\bar{k}$ are compared in Fig. 6(b) for the default setting. Since the slave is stiff and rough, viscous dissipation is only sustained in the continuously indented master, which can also be observed from the internal variable distribution in Fig. 5(a). The total normalized macroscopic dissipation delivers a $\overline{\mathrm{k}}_{d}$ value that accurately matches $\overline{\mathbb{k}}_{m}$, as predicted by (3.15). Figs. 6(c) and (d) summarize the remaining thermomechanical contact quantities. The microscopic temperature jump $\vartheta_{c}$ is negligible due to the large value of contact conductivity $k_{c}$ employed, which essentially acts as a penalty parameter. Its macroscopic counterpart $\bar{\vartheta}_{c}$, on the other hand, has a large value due to the rough contact interface topography (Temizer, 2014a). Moreover, the interface topography depends on the material response, for instance a smaller jump is obtained for purely elastic bodies. However, unlike $\overline{\mathbb{k}}_{m}$, the absence of microscopic friction does not appreciably alter the value of $\bar{\vartheta}_{c}$. Finally, the microscopic relative effusivities are fixed at a value of 0.5 . In the absence of viscous dissipation, the macroscopic values are the same. However, in the default setting that includes viscous dissipation, these partitioning constants are significantly different. Specifically, when microscopic friction is not present then $\bar{\gamma}^{(2)}=1$, that is the frictional heating on the macroscale is transmitted entirely to the slave as predicted in Section 3.3.3.

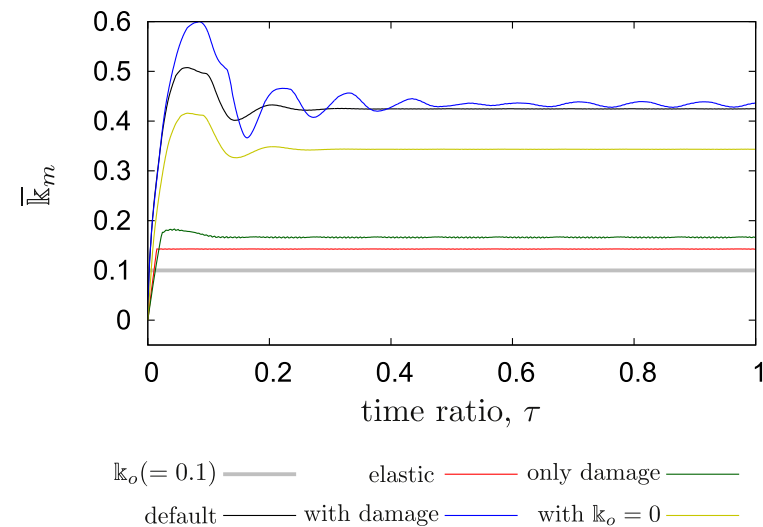

(a) macroscopic friction coefficient

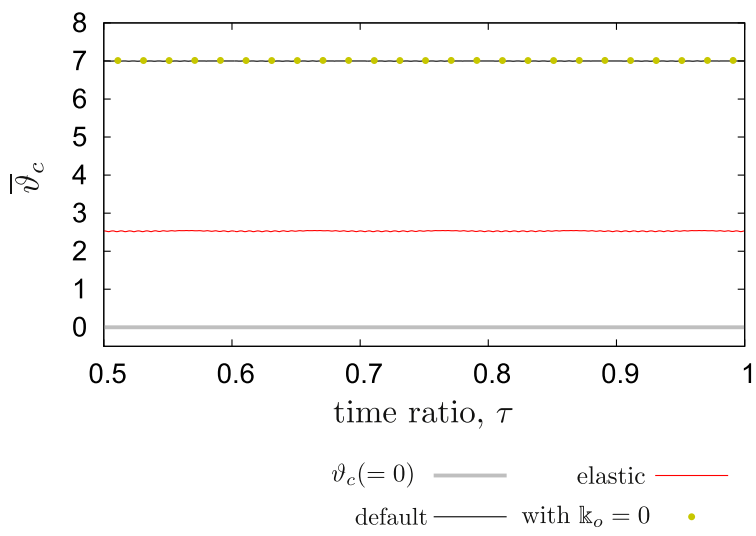

(c) macroscopic temperature jump
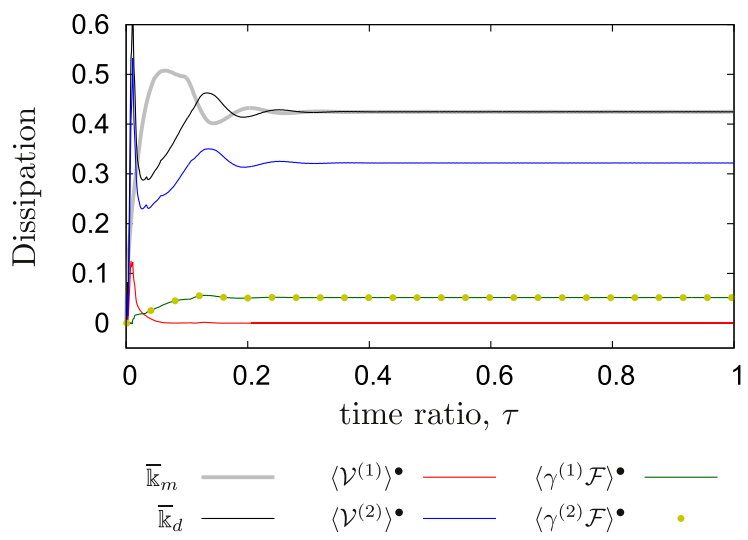

(b) normalized macroscopic dissipation

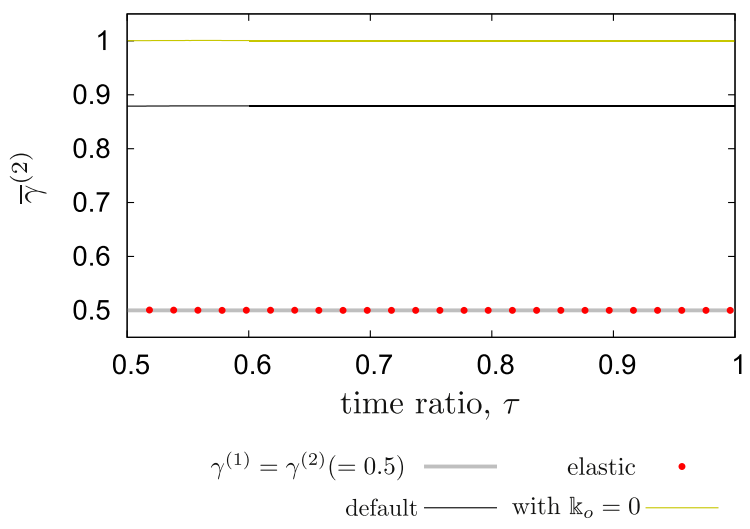

(d) macroscopic relative effusivity

Fig. 6. Effect of microscopic bulk and interface modeling choices are summarized. 


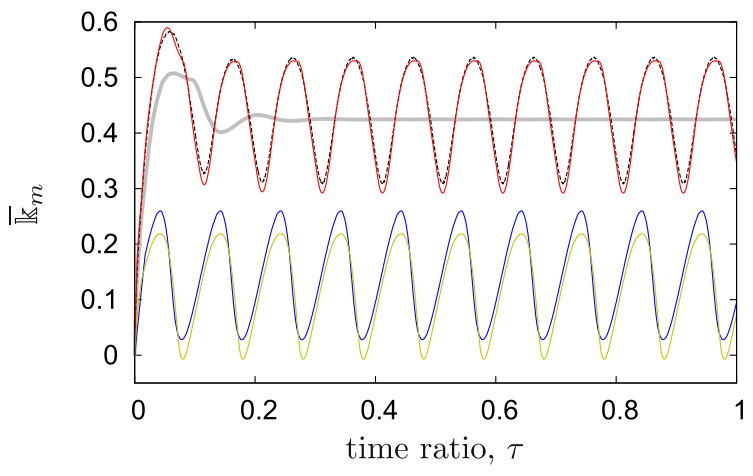

both rough $\longrightarrow \quad$ elastic $\longrightarrow \quad 1 \longleftrightarrow 2$

(a) macroscopic friction coefficient

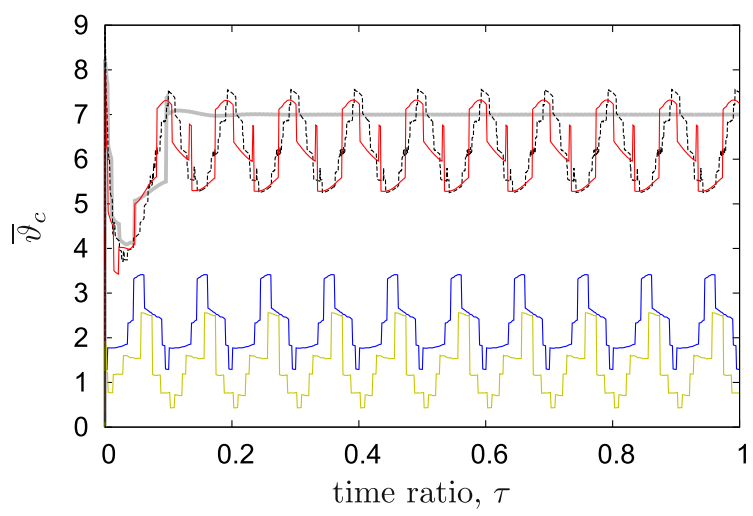

(c) macroscopic temperature jump

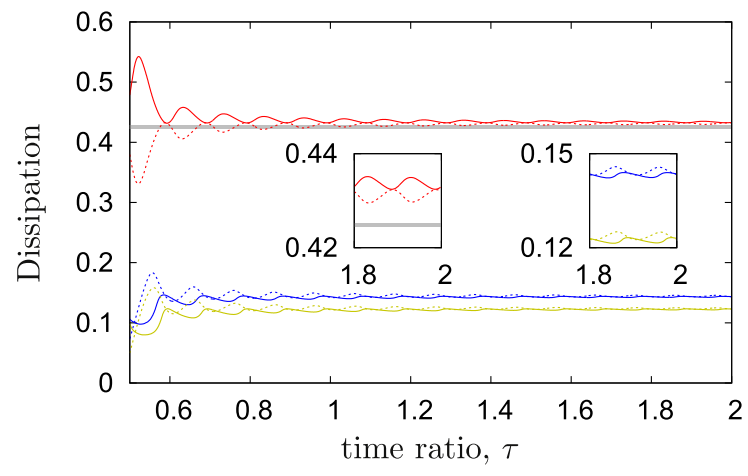

$\overline{\mathbb{k}_{m}}$

(b) normalized macroscopic dissipation

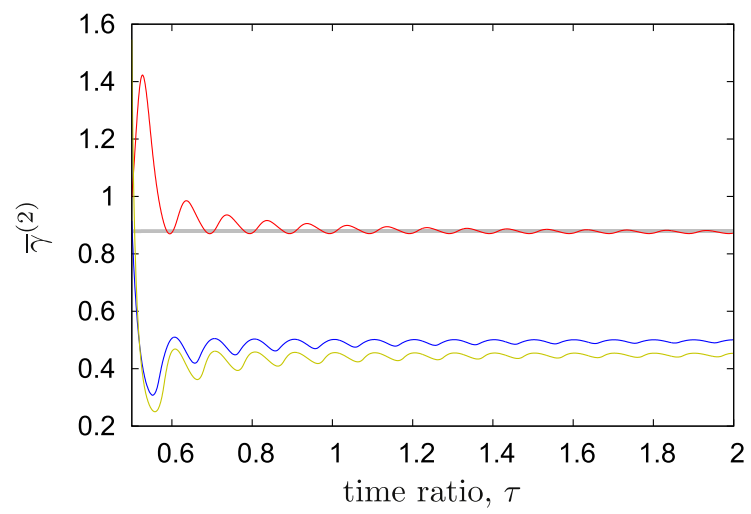

(d) macroscopic relative effusivity

Fig. 7. In (a), the red case indicates $\bar{k}$ in the case of bilateral roughness where the master is assigned an RMS value of $\sigma^{(2)}=\sigma^{(1)} / 7.5$. The default unilateral roughness result is the gray case. The blue case omits viscous dissipation and the yellow case switches the RMS values of the slave and the master, both cases reflecting the changes relative to the red one. Plots (b)-(d) retain the same legend. In (b), the time-averaged values of $\bar{k}_{d}$ and $\bar{k}_{m}$ are additionally compared for each case. Finally, in plots (a) and (c), the default case is re-computed with higher space-time resolution (by using half the default time step size and six times the default number of elements in each direction) and the results are indicated with a dotted black line. (For interpretation of the references to color in this figure caption, the reader is referred to the web version of this paper.)

\subsection{Interface topography and macroscopic contact parameters}

In the default setting where only the slave is rough, macroscopic friction is not oscillatory in time. In the case of bilateral roughness (Fig. 4), significant oscillations are observed as summarized in Fig. 7. These oscillations depend on the material response, and the topography of each surface explicitly influences the macroscopic friction which is demonstrated by switching the roughness assignments. In all cases, the mechanical and dissipation-based definitions of friction match closely in a time-averaged sense as expected. In all remaining investigations, only $\overline{\mathbb{k}}_{m}$ will be monitored. The macroscopic relative

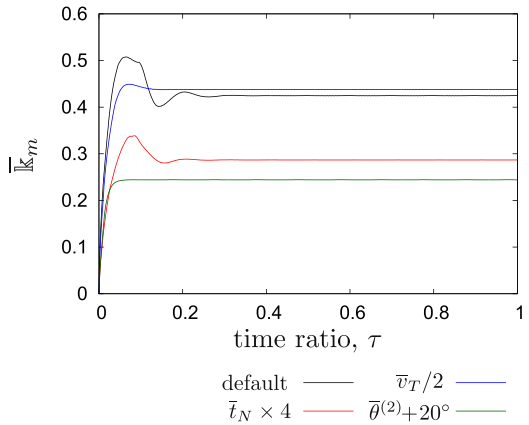

(a) macroscopic friction coefficient

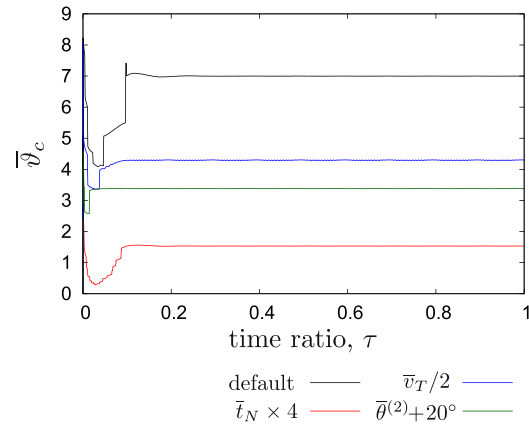

(b) macroscopic temperature jump

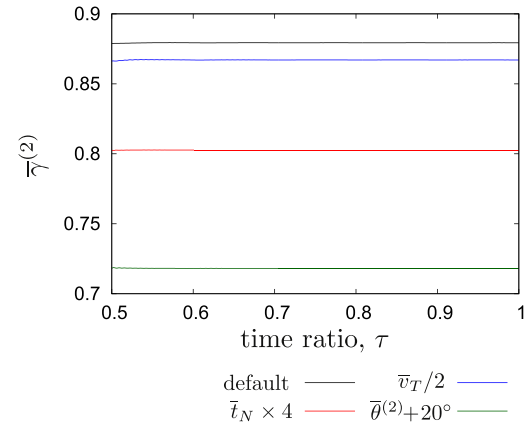

(c) macroscopic relative effusivity

Fig. 8. Effect of varying primary macroscopic contact parameters is summarized. 
effusivity definition (3.27) intrinsically involves time-averaging so that these quantities converge to constant values, provided sufficiently long dragging durations are chosen. Finally, strong oscillations are also observed in the macroscopic temperature jump. Here, the non-smoothness in the evolution of $\bar{\vartheta}_{c}$ is due to the sensitivity of the thermal contact algorithm employed to the contact active set which continuously changes during sliding. Nevertheless, further increasing the spacetime resolution from the default choices gradually smoothens these oscillations while causing little change in the amplitude and the mean, which is demonstrated in Figs. 7(a) and (c) for $\overline{\mathrm{k}}_{m}$ and $\bar{\vartheta}_{c}$, respectively. Consequently, the trends observed at the default resolution are already representative of the macroscopic thermomechanical interface response and highlight the complexities of dealing with bilateral roughness. In Fig. 7(d), the overshoot beyond the theoretically maximum value of one is due to the fact that, instead of the explicit expression (3.27) 2 , (3.27) ${ }_{1}$ has been employed with $\overline{\mathcal{F}}$ from (3.15) 1 , which initially underestimates (3.15) 2 (see Fig. 7(b)). Since only converged time averages are meaningful macroscopically, this transient behavior may be omitted.

For a given topography of the microscopic interface as well as models for the bulk and interface behavior, the primary macroscopic contact variables $\left\{\bar{t}_{N}, \overline{\boldsymbol{v}}_{T}, \bar{\theta}^{(2)}, \bar{h}^{c}\right\}$ control the dual ones $\left\{\overline{\mathrm{k}}, \bar{\vartheta}_{c}, \bar{\gamma}^{(2)}\right\}$. The link between the two sets is partially demonstrated in Fig. 8 based on the default simulation setting. In addition to the well-known temperature-velocitypressure dependence of $\bar{k}$, it is interesting to observe a similar dependence for $\bar{\vartheta}_{c}$ and $\bar{\gamma}^{(2)}$. However, although significant effort has been made towards modeling this dependence of $\bar{k}$, for instance through rate- and state-dependent friction models (Ronsin and Coeyrehourcq, 2001; Putelat et al., 2010; Woodhouse and Wang, 2011), a comparable effort for $\bar{\vartheta}_{c}$ and $\bar{\gamma}^{(2)}$ is missing in the literature.

\subsection{Numerical parameters and choices}

The investigations are continued with a series of remarks regarding the numerical effect of simulation parameters and algorithmic choices. Among the simulation parameters, the time step size plays an important role in assessing the validity of micro-macro dissipation equalities. Specifically, a non-zero gap can remain between $\overline{\mathrm{k}}_{m}$ and $\overline{\mathrm{k}}_{d}$ even after time averaging which, for instance, is visible in Fig. 7(b). One reason is the various simplifications carried out during derivation, some of which were discussed in Section 5.1.3. Among these, the non-dissipative heating term is monitored in Fig. 9 for the default setting. Since only the master is subjected to cyclic loading during sliding, the heating term in the slave eventually converges to zero. For the master, the elastic and viscous portions of the heating term in (5.14) are individually non-zero but their sum is expected to vanish. The sum is observed to be non-zero yet already small compared to $\bar{k}$ in magnitude for the default time step size. Clearly, finer time step sizes result in a smaller error and hence would lead to a better agreement between the mechanical and dissipation-based definitions of friction and, thereby, to a numerically more accurate prediction of all macroscopic thermomechanical contact parameters.

All the results presented so far were based on the incrementally two-phase micromechanical test of Section 4.2 where an explicit temperature enforcement is proposed. If dissipative heating is reflected in the thermal energy balance during the computations, the temperature in the samples continuously rise during sliding. Through the strong temperature dependence of the thermo-viscoelastic material model, this rise reflects onto the overall dissipation and the contact interface topography, thereby leading to a continuously varying macroscopic thermomechanical contact response for this coupled approach as summarized in Fig. 10. On the one hand, this observation raises the question of whether there is truly a scale separation for the problem at hand with respect to space and time. On the other hand, it highlights the advantage of the two-phase test, namely that a well-defined macroscopic response is obtained independent of the duration of sliding. Hence, the two-phase approach is a convenient first step towards the characterization of the complex thermomechanical contact response on the macroscale.

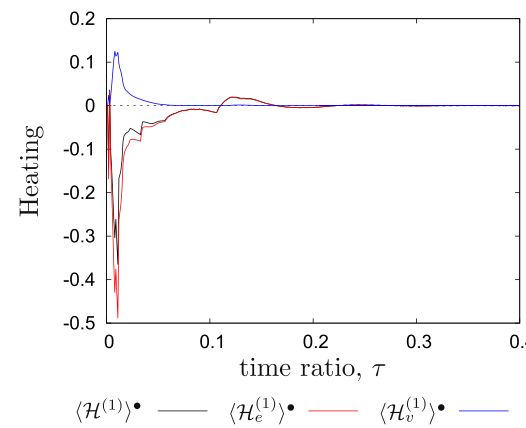

(a) normalized heating in $\mathcal{C}_{O}^{(1)}$

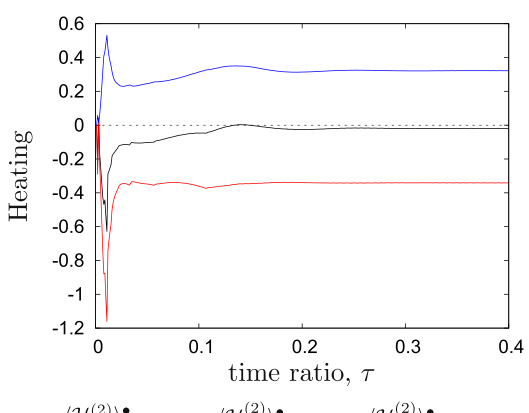

$\left\langle\mathcal{H}^{(2)}\right\rangle^{\bullet}-\left\langle\mathcal{H}_{e}^{(2)}\right\rangle^{\bullet}\left\langle\mathcal{H}_{v}^{(2)}\right\rangle^{\bullet}$

(b) normalized heating in $\mathcal{C}_{o}^{(2)}$

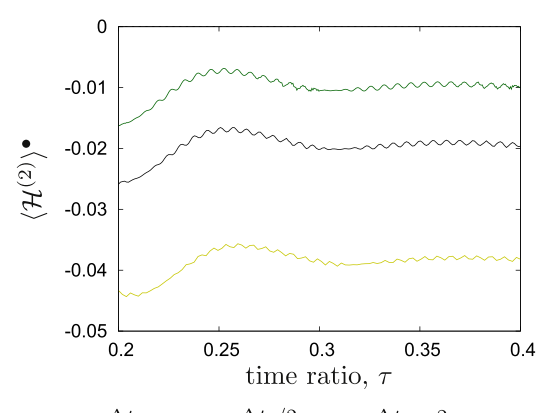

$\Delta t_{o}-\Delta t_{o} / 2-\Delta t_{o} \times 2$

(c) effect of time step size

Fig. 9. The non-dissipative heating terms of (5.14) are monitored. 


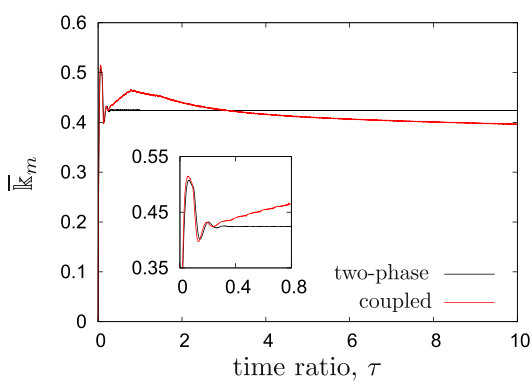

(a) macroscopic friction coefficient

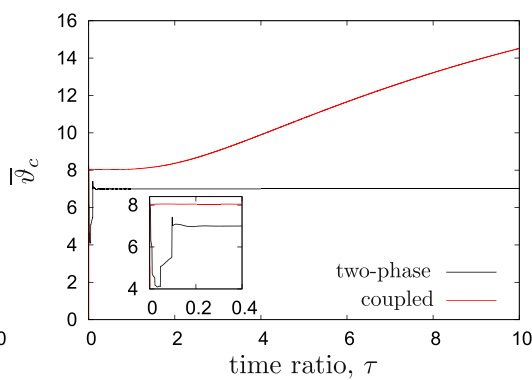

(b) macroscopic temperature jump

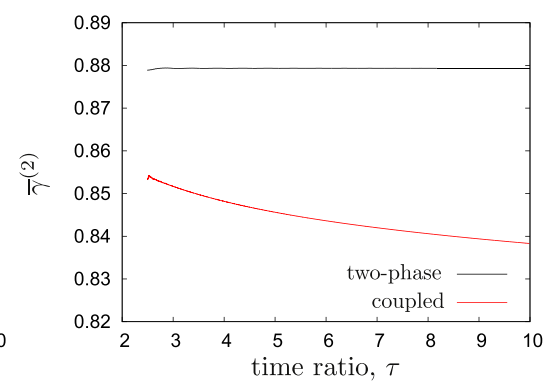

(c) macroscopic relative effusivity

Fig. 10. Contrary to the two-phase micromechanical test framework employed in earlier sections, dissipative heating is reflected in the thermal energy balance to illustrate the resulting time variation in the macroscopic response.

\subsection{Random roughness}

The homogenization framework can accommodate three-dimensional random roughness (Temizer, 2014b). Presently, only two-dimensional bilateral random roughness will be addressed based on the parameters for its periodic counterpart but by reducing the RMS roughness on both surfaces by a factor of two. For each sample size, ranging from a length of $L_{o}$ to $5 L_{0}$, 20 sample realizations are tested (Fig. 11). A dragging time of $1.5 T_{o}$ is employed for all samples (see Table 1 ), except for the smallest size for which $3 T_{o}$ was chosen to ensure convergence of the moving time average.

The histories of the macroscopic friction coefficient and temperature jump are summarized in Fig. 12, which display the significant scatter in the sample response at small sizes. Despite this scatter, the average response from multiple realizations is of comparable magnitude at different sample sizes. Such a comparison is explicitly demonstrated in Fig. 13 for the macroscopic relative effusivity where the advantage of employing a large sample size, namely a smaller standard deviation in sample responses, is additionally observed.

The macroscopic contact response of random samples is also strongly temperature-velocity-pressure dependent. Concentrating on dissipation partitioning, this dependence will be demonstrated by varying the macroscopic contact pressure $\bar{t}_{N}$ and the glass transition temperature $\theta_{g}$. Note that the effect of the variation of $\theta_{g}$ is relative to the values of the

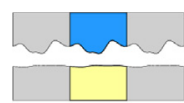

(a) size $=1$

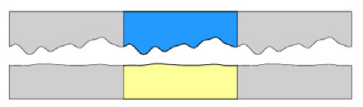

(b) $\operatorname{size}=2$

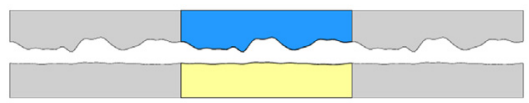

(c) size $=3$

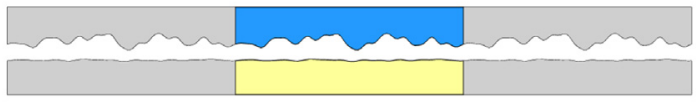

(d) $\operatorname{size}=4$

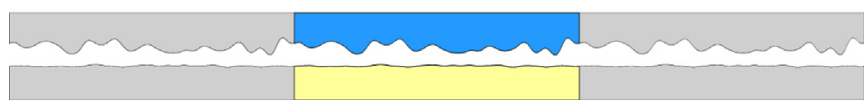

(e) size $=5$

Fig. 11. Single bilateral random roughness realizations for the different sample sizes employed. The size factor multiplied by the default length $L_{o}$ (Table 1 ) delivers the actual length of the sample. Although the roughness on the master surface is small, it is sufficient to induce significant oscillations in the macroscopic response (Fig. 12). 


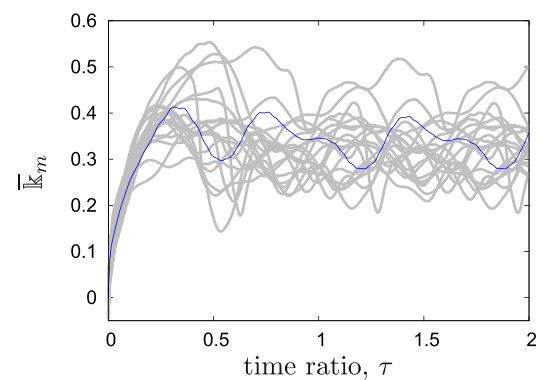

$(\mathrm{a}-1)$ size $=1$

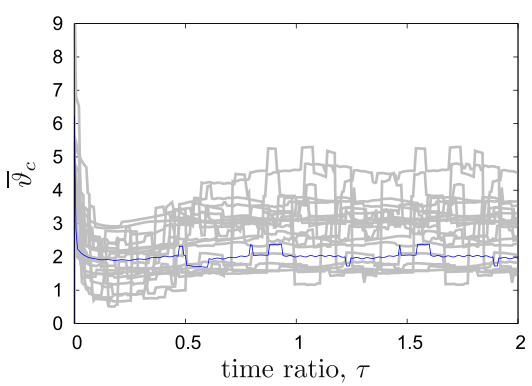

(b-1) size $=1$

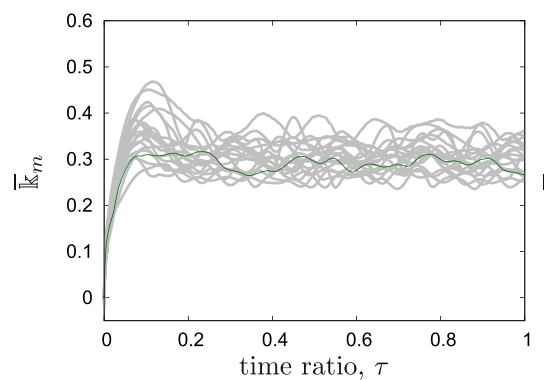

(a-2) size $=3$

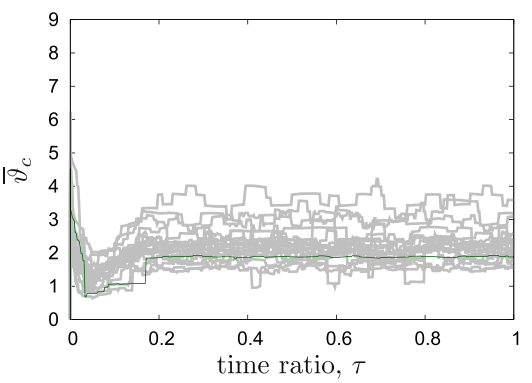

(b-2) size $=3$

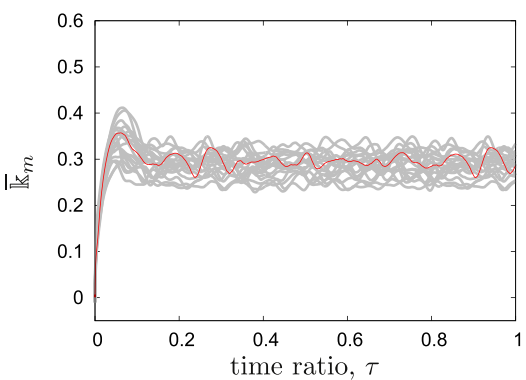

$(\mathrm{a}-3)$ size $=5$

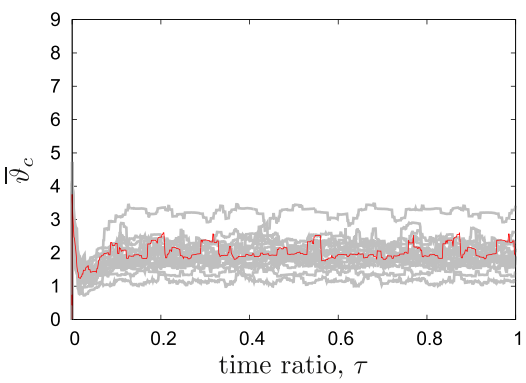

(b-3) size $=5$

Fig. 12. The macroscopic friction coefficient and temperature jump histories $\left(\tau=t / 1.5 T_{o}\right.$ ) for 20 sample realizations at selected sample sizes (see Fig. 11 ). The lines with color indicate the particular response corresponding to the sample displayed in Fig. 11.

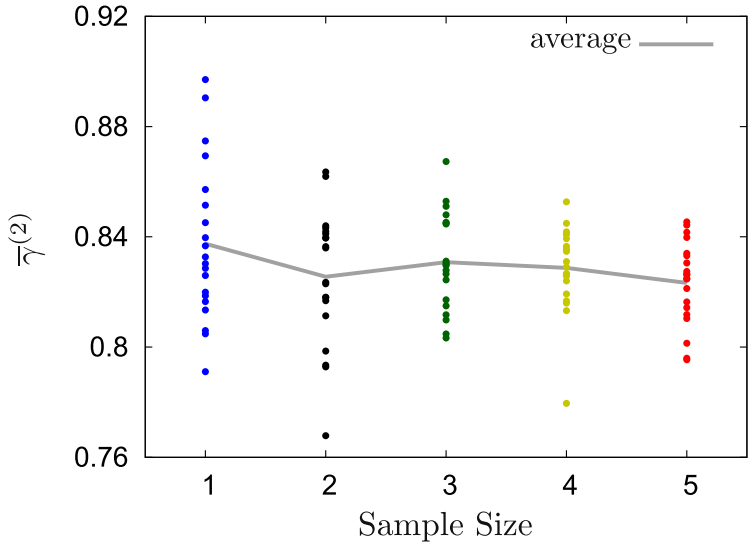

(a) response for different realizations

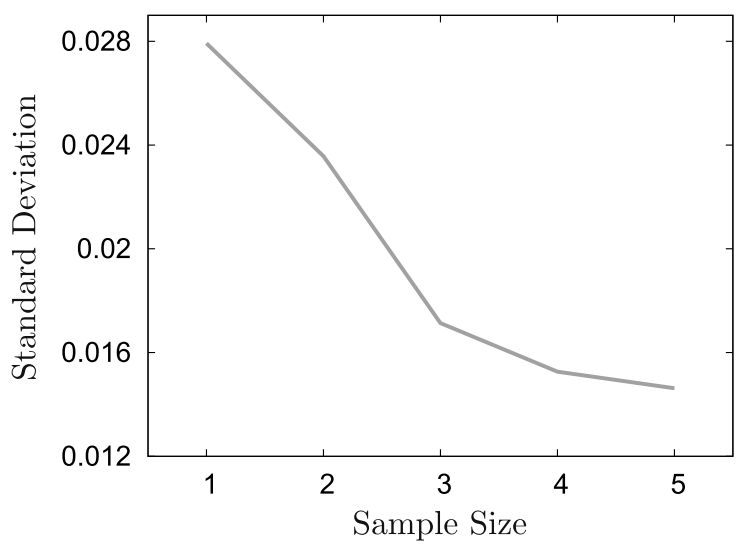

(b) scatter in sample response

Fig. 13. Macroscopic relative effusivity at different sample sizes.

macroscopic surface temperatures. Moreover, when $\theta_{g}$ is varied the major material parameter that is affected is the viscosity, the influence of which is relative to the slip velocity. Hence, the temperature-velocity dependence of the macroscopic contact response is implicit in the dependence on $\theta_{g}$. Results for a single random sample are summarized in Fig. 14. Here, in order to monitor the individual contributions to dissipation from the bulk and the interface, (3.27) is decomposed as

$$
\bar{\gamma}_{\mathcal{F}}^{(I)}=\frac{\left\langle\gamma^{(I)} \mathcal{F}\right\rangle}{\overline{\mathcal{F}}}, \quad \bar{\gamma}_{\mathcal{V}}^{(I)}=\frac{\left\langle\mathcal{V}^{(I)}\right\rangle^{\searrow}}{\overline{\mathcal{F}}} .
$$

Clearly, different contributions display independent trends although the bulk dissipation dictates the overall behavior for this example. 


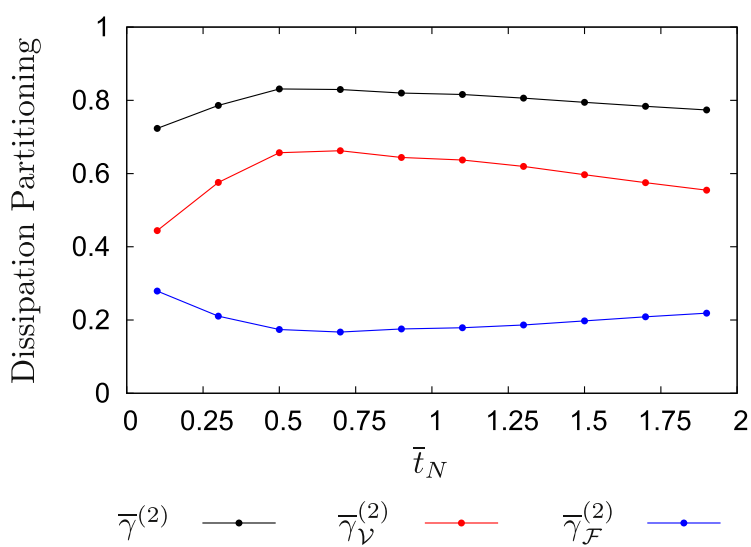

(a) macroscopic contact pressure $\bar{t}_{N}$ is varied

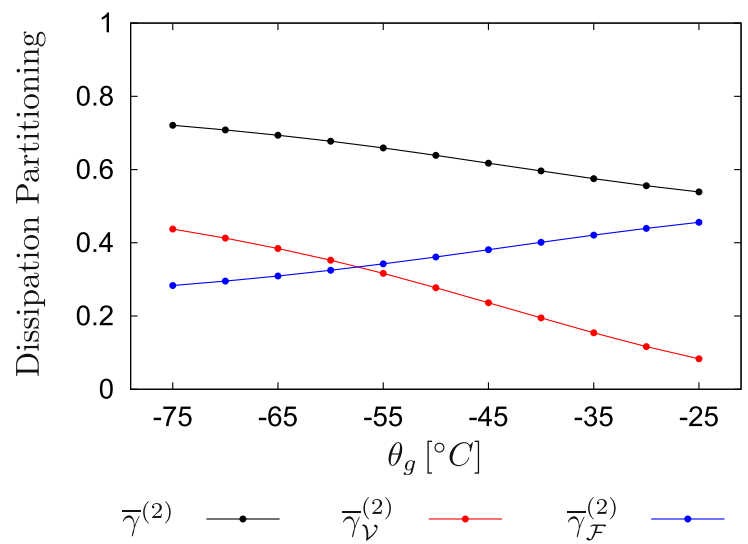

(b) glass transition temperature $\theta_{g}$ is varied

Fig. 14. Variations of the macroscopic effusivity and the individual contributions from (6.4) are monitored with respect to the macroscopic contact pressure and the glass transition temperature. Here, the random sample from Fig. 11(c) has been employed and the surface temperatures are elevated to $\theta_{0}+25$ from their default value $\theta_{0}$.

\section{Conclusion}

A homogenization framework has been presented for determining the macroscopic thermomechanical sliding contact response of soft interfaces with microscopic roughness. In addition to the friction coefficient, the complete characterization of this response entails the determination of the temperature jump across the macroscopic contact interface as well as the partitioning coefficients which are associated with the frictional dissipation as it is perceived on the macroscale. Towards this purpose, mechanical and thermal micro-macro dissipation equalities were derived and subsequently employed within an incrementally two-phase micromechanical test where the contact surface temperatures are explicitly enforced to obtain a well-defined macroscopic interface response. The overall framework is first-order in the sense that it is motivated by a separation of length and time scales between the macroscopic contact problem and the micromechanical test problem. The computational implementation of the framework was carried out in a recently developed periodically embedded micromechanical setting that is suitable for the simulation of two deformable samples in large sliding contact.

The numerical results presented already highlight the macroscopic tribological complexity associated with soft interfaces in a qualitative manner, where not only the friction coefficient but also the temperature jump and the partitioning coefficients are observed to be temperature-velocity-pressure dependent. Nevertheless, a quantitatively more accurate assessment of the thermomechanical interactions between the macroscopic contact response and the microscopic physics requires addressing a number of simplifications that have been assumed in the numerical investigations from a physical perspective. These include the omission of inertial effects and interface adhesion, the assumption of a single scale of roughness and a single relaxation time, the absence of bulk heterogeneities in the boundary layer or a fluid film at the contact interface, among others. All of these features are known to play an important role in the macroscopic sliding contact response and an investigation of their influence in a three-dimensional setting is a future goal. From a numerical perspective, despite the potential of the framework to address complex interface topographies, a number of shortcomings need to be assessed. For instance, the numerical setup can presently admit only matching macroscopic stretches on the slave and master surfaces. Significantly differing finite stretches are particularly relevant for soft interfaces in three dimensions. Additionally, the method of incorporating random roughness may not be optimal since it requires partial modification of the original topography to ensure periodic embedding. Nevertheless, the overall homogenization framework appears to be a viable starting point to address the challenging micromechanics of soft interfaces. Efficient theoretical and numerical approaches that are often available for infinitesimally deforming interfaces are to a large extent not applicable to soft interfaces in large sliding contact. The development of effective frameworks for these interfaces will be an ongoing pursuit.

\section{Acknowledgements}

Support for this work was provided by the Scientific and Technological Research Council of Turkey (TÜBITAK) under the Career Programme Grant no. 110M661.

\section{Appendix A. Constitutive modeling}

In this appendix, further details of the material model outlined in Section 5.1 are provided. The parameter-normalized functions appearing in the elastic and viscous contributions of (5.5) and (5.6) are chosen to be the same for both branches: 


$$
\mathcal{E}_{x}(\zeta)=\ln \zeta, \quad \Psi_{x}^{\mathrm{vol}}(\zeta)=(\zeta-\ln \zeta-1) / 4, \quad \Psi_{x}^{\mathrm{dev}}(\boldsymbol{B})=\sum_{p=1}^{M} \frac{\gamma_{p}}{\beta_{p}}\left(\sum_{i=1}^{d} \Lambda_{i}^{\beta_{p} / 2}-d\right)
$$

Here, the deviatoric part represents an Ogden material in a $d$-dimensional setting with $\Lambda_{i}$ as the eigenvalues of its argument

B. The heat capacity functions in (5.3) also have the same form:

$$
g_{x}(\theta)=b\left(\theta / \theta_{0}\right)^{a} .
$$

Damage variables $G_{x}$ evolve via (5.8) according to the model of Simo (1987) depending on the history of

$$
\Xi_{x}=\sqrt{2 \bar{\psi}_{x}^{\mathrm{dev}}}
$$

$G_{x}$ monotonically decreases to a saturation value $G_{\infty x}$ at a rate that is controlled by a prescribed value. Details (Temizer and Wriggers, 2010a) are omitted since damage is of minor importance in the present work.

Following Reese and Govindjee (1998b), the viscous evolution is based on (5.11) and the Lie derivative $\mathcal{L}\left[\boldsymbol{b}_{e}\right]$ :

$$
-\frac{1}{2} \mathcal{L}\left[\boldsymbol{b}_{e}\right] \boldsymbol{b}_{e}^{-1}=\frac{1}{2 \eta} \tau_{v}^{\operatorname{dev}}
$$

This ensures the non-negativity of the viscous contribution to the mechanical dissipation

$$
\mathcal{V}=-\tau_{v}^{\operatorname{dev}} \cdot \frac{1}{2} \mathcal{L}\left[\boldsymbol{b}_{e}\right] \boldsymbol{b}_{e}^{-1} \geq 0
$$

while monotonically decreasing $G_{x}$ ensure the non-negativity of the damage contribution

$$
\mathcal{D}=-\bar{\psi}_{e}^{\mathrm{dev}} \dot{G}_{e}-\bar{\psi}_{v}^{\mathrm{dev}} \dot{G}_{v} \geq 0
$$

so that the overall mechanical dissipation $\mathcal{M}=\mathcal{V}+\mathcal{D}$ is guaranteed to be non-negative.

\begin{tabular}{|c|c|c|c|}
\hline \multirow[t]{2}{*}{ Composite sample geometry } & $\mu \mathrm{m}$ & $L_{o}$ & 10 \\
\hline & & $H_{o}^{(I)}$ & $0.6 L_{0}$ \\
\hline \multirow[t]{2}{*}{ RMS roughness } & & $\sigma^{(1)}$ & $3 L_{0} / 20$ \\
\hline & & $\sigma^{(2)}$ & 0 \\
\hline Macroscopic contact pressure & bar & $\bar{t}_{N}$ & 10 \\
\hline Macroscopic slip velocity magnitude & $\mathrm{m} / \mathrm{s}$ & $\bar{v}_{T}$ & 10 \\
\hline Macroscopic contact heat flux & $\mathrm{W} / \mathrm{mm}^{2}$ & $\bar{h} c$ & 1 \\
\hline Nominal dragging time & & $T_{o}$ & $L_{0} / \bar{v}_{F}$ \\
\hline \multirow[t]{2}{*}{ Time averaging parameters } & & $t_{o}$ & $5 T_{o}$ \\
\hline & & $T_{\text {avg }}$ & $5 T_{o}$ \\
\hline Nominal time step size & & $\Delta t$ & $10^{-2} T_{0}$ \\
\hline \multirow[t]{2}{*}{ Number of elements (quartic B-splines) } & & $N_{X}$ & 15 \\
\hline & & $N_{Y}$ & 9 \\
\hline \multirow[t]{4}{*}{ Linear elasticity parameters } & $\mathrm{MPa}$ & $\mu_{\mathrm{oe}}$ & 5 \\
\hline & & $\mu_{\mathrm{ov}}$ & $5 \mu_{o e}$ \\
\hline & & $\kappa_{o e}$ & $12 \mu_{o e}$ \\
\hline & & $\kappa_{o v}$ & $12 \mu_{o v}$ \\
\hline Initial (reference) temperature & ${ }^{\circ} \mathrm{C}$ & $\theta_{0}$ & 20 \\
\hline Glass transition temperature & ${ }^{\circ} \mathrm{C}$ & $\theta_{g}$ & -50 \\
\hline Shear viscosity at $\theta_{0}$ & & $\eta$ & $\left(10^{-2} T_{o}\right) \mu_{\mathrm{ov}}$ \\
\hline Macroscopic surface temperatures & & $\bar{\theta}^{(I)}$ & $\theta_{0}$ \\
\hline Linear thermal expansion coefficient & $\mathrm{K}^{-1}$ & $\alpha_{\mathrm{ox}}$ & $10^{-5}$ \\
\hline Specific heat per unit volume & $\mathrm{MPa} / \mathrm{K}$ & $c_{o}$ & 1.74 \\
\hline \multirow[t]{2}{*}{ Thermal conductivity } & $\mathrm{W} / \mathrm{m} \mathrm{K}$ & $k_{o}$ & 1 \\
\hline & $\mathrm{W} / \mathrm{m} \mathrm{K}^{2}$ & $k_{1}$ & 0 \\
\hline Relative effusivities & - & $\gamma^{(1)}$ & 0.5 \\
\hline \multirow[t]{2}{*}{ Thermal contact parameters } & $\mathrm{W} / \mathrm{mm}^{2} \mathrm{~K}$ & $k_{c}$ & 10 \\
\hline & - & $\alpha$ & 1 \\
\hline Damage variable saturation & - & $G_{\infty X}$ & 0.2 \\
\hline
\end{tabular}

Finally, the temperature dependence of $\eta$ is incorporated through the time-temperature equivalence principle combined with the Williams-Landel-Ferry model based on the relative temperature $\vartheta_{g}$ with respect to the glass transition

Table 1

Default simulation parameters. Only the changes to these default choices will be explicitly noted. The material parameters are for the slave $\left(\mathcal{B}^{(1)}\right)$. For the master $\left(\mathcal{B}^{(2)}\right), \mu_{o e}^{(2)}=\mu_{o e}^{(1)} / 8$. All remaining parameters have the same values or calculated similarly. 
temperature $\theta_{g}$ of the material (Ward and Sweeney, 2004):

$$
\vartheta_{g}=\theta-\theta_{g} \longrightarrow \log a=-17.4 \vartheta_{g} /\left(51.6+\vartheta_{g}\right) \longrightarrow \eta(\theta) / \eta\left(\theta_{g}\right)=a .
$$

\section{Appendix B. Simulation parameters}

Table 1 lists the default values of various simulation parameters which are subject to further adjustment throughout the numerical investigations. The Ogden material parameters $\left\{\gamma_{p}, \beta_{p}\right\}$ are chosen according to Reese and Govindjee (1998b) (not shown) and several thermal properties according to Reese and Govindjee (1998a).

\section{References}

Adams, M.J., Johnson, S.A., Lefèvre, P., Lévesque, V., Hayward, V., André, T., Thonnard, J.-L., 2013. Finger pad friction and its role in grip and touch. J. R. Soc. Interface 10, 20120467.

Agelet de Saracibar, C., 1999. Numerical analysis of coupled thermomechanical frictional contact problems. Computational model and applications. Arch. Comput. Methods Eng. 5, 243-301.

Alart, P., Curnier, A., 1991. A mixed formulation for frictional contact problems prone to Newton like solution methods. Comput. Methods Appl. Mech. Eng. 92, 353-375

Bahrami, M., Culham, J.R., Yovanovich, M.M., Schneider, G.E., 2006. Review of thermal joint resistance models for nonconforming rough surfaces. Appl. Mech. Rev. 59, 1-12.

Bai, Y., Jagota, A., Hui, C.-Y., 2014. Frictional auto-roughening of a surface with spatially varying stiffness. Soft Matter 10, $2169-2177$.

Borri, C., Paggi, M., 2015. Topological characterization of antireflective and hydrophobic rough surfaces: are random process theory and fractal modeling applicable?. J. Phys. D: Appl. Phys. 48, 045301.

Bowden, F.B., Tabor., D., 2008. The Friction and Lubrication of Solids. Oxford University Press, New York.

Brörmann, K., Barey, I., Urbakh, M., Bennewitz, R., 2013. Friction on a microstructured elastomer surface. Tribol. Lett. 50, 3-15.

Carbone, G., Putignano, C., 2013. A novel method to predict sliding and rolling friction of viscoelastic materials: theory and experiments. J. Mech. Phys. Solids $61,1822-1834$.

Carbone, G., Lorenz, B., Persson, B.N.J., Wohlers, A., 2009. Contact mechanics and rubber friction for randomly rough surfaces with anisotropic statistical properties. Eur. Phys. J. E 29, 275-284.

Chadwick, P., 1974. Thermo-mechanics of rubberlike materials. Philos. Trans. R. Soc. Lond. Ser. A 276, 372-403.

Chadwick, P., Creasy, C.F.M., 1984. Modified entropic elasticity of rubberlike materials. J. Mech. Phys. Solids 32 (5), 337-357.

Chantrenne, P., Raynaud, M., 1997. A microscopic thermal model for dry sliding contact. Int. J. Heat Mass Transf. 40, 1083-1094.

Chantrenne, P., Raynaud, M., 2001. Study of a macroscopic sliding contact thermal model from microscopic models. Int. J. Therm. Sci. 40, 603-621.

Ciavarella, M., Demelio, G., Barber, J.R., Jang, Y.H., 2000. Linear elastic contact of the Weirstrass profile. Proc. R. Soc. Lond. A 456, $387-405$.

De Lorenzis, L., Wriggers, P., 2013. Computational homogenization of rubber friction on rough rigid surfaces. Comput. Mater. Sci. 77, 264-280.

Fagiani, R., Massi, F., Chatelet, E., Costes, J.P., Berthier, Y., 2012. Contact of a finger on rigid surfaces and textiles: Friction coefficient and induced vibrations. Tribol. Lett. 48, 145-158.

Grosch, K.A., 1963. The relation between the friction and visco-elastic properties of rubber. Proc. R. Soc. Lond. A 274, 21-39.

Gurtin M.E., Fried E., Anand L., 2010. The Mechanics and Thermodynamics of Continua, Cambridge.

Holzapfel, G.A., 2001. Nonlinear Solid Mechanics: A Continuum Approach for Engineering. Wiley, Chichester.

Hughes, T.J.R., Cottrell, J.A., Bazilevs, Y., 2005. Isogeometric analysis: CAD, finite elements, NURBS, exact geometry and mesh refinement. Comput. Methods Appl. Mech. Eng. 194, 4135-4195.

Jones, R.E., Papadopoulos, P., 2006. Simulating anisotropic frictional response using smoothly interpolated traction fields. Comput. Methods Appl. Mech. Eng. 195, 588-613.

Klüppel, M., Heinrich, G., 2000. Rubber friction on self-affine road tracks. Rubber Chem. Technol. 73, 578-606.

Koç, İ.M., Aksu, C., 2013. Tactile sensing of constructional differences in fabrics with a polymeric finger tip. Tribol. Int. 59, 339-349.

Konyukhov, A., Vielsack, P., Schweizerhof, K., 2008. On coupled models of anisotropic contact surfaces and their experimental validation. Wear 264 $579-588$.

Laraqi, N., 1997. Velocity and relative contact size effects on the thermal constriction resistance in sliding solids. J. Heat Transf. 119, 173-177.

Laursen, T.A., 2003. Computational Contact and Impact Mechanics, 1st ed Springer, Berlin, Heidelberg, New York (corr. 2nd printing).

Le Gal, A., Klüppel, M., 2008. Investigation and modelling of rubber stationary friction on rough surfaces. J. Phys.: Condens. Matter $20,015007$.

Lion, A., 1997. On the large deformation behavior of reinforced rubber at different temperatures. J. Mech. Phys. Solids 45, 1805-1834.

Madhusudana, C.V., 1996. Thermal Contact Conductance. Springer, New York.

McBride, A., Mergheim, J., Javili, A., Steinmann, P., Bargmann, S., 2012. Micro-to-macro transitions for heterogeneous material layers accounting for in-plane stretch. J. Mech. Phys. Solids 60, 1221-1239.

Miehe, C., Keck, J., 2000. Superimposed finite elastic-viscoelastic-plastoelastic stress response with damage in filled rubbery polymers: experiments, modelling and algorithmic implementation. J. Mech. Phys. Solids 48, 323-365.

Momozono, S., Takeuchi, H., Iguchi, Y., Nakamura, K., Kyogoku, K., 2012. Dissipation characteristics of adhesive kinetic friction on amorphous polymer surfaces. Tribol. Int. 48, 122-127.

Murarash, B., Itovich, Y., Varenberg, M., 2011. Tuning elastomer friction by hexagonal surface patterning. Soft Matter 7, 5553-5557.

Nosko, O., 2013. Partition of friction heat between sliding semispaces due to adhesion-deformational heat generation. Int. J. Heat Mass Transf. 64 $1189-1195$.

Paggi, M., Ciavarella, M., 2010. The coefficient of proportionality $\kappa$ between real contact area and load, with new asperity models. Wear 268, $1020-1029$.

Pei, L., Hyun, S., Molinari, J.F., Robbins, M.O., 2005. Finite element modeling of elasto-plastic contact between rough surfaces. J. Mech. Phys. Solids 53 , 2385-2409.

Persson, B.N.J., 2000. Sliding Friction, 2nd edition. Springer-Verlag, Berlin, Heidelberg, New York.

Persson, B.N.J., 2001. Theory of rubber friction and contact mechanics. J. Chem. Phys. 115, 3840-3861.

Persson, B.N.J., 2014. Role of frictional heating in rubber friction. Tribol. Int. 56, 77-92.

Persson, B.N.J., Albohr, O., Tartaglino, U., Volokitin, A.I., Tosatti, E., 2005. On the nature of surface roughness with applications to contact mechanics, sealing, rubber friction and adhesion. J. Phys.: Condens. Matter 17, R1-R62.

Piegl, L., Tiller, W., 1996. The NURBS Book, 2nd edition. Springer, Berlin, Heidelberg, New York.

Putelat, T., Dawes, J.H.P., Willis, J.R., 2010. Regimes of frictional sliding of a spring-block system. J. Mech. Phys. Solids 58, 27-53.

Ramalho, A., Szekeres, P., Fernandes, E., 2013. Friction and tactile perception of textile fabrics. Tribol. Int. 63, 29-33. 
Reese, S., Govindjee, S., 1998a. Theoretical and numerical aspects in the thermo-viscoelastic material behavior of rubber-like polymers. Mech. TimeDepend. Mater. 1, 357-396.

Reese, S., Govindjee, S., 1998b. A theory of finite viscoelasticity and numerical aspects. Int. J. Solids Struct. 35 (26-27), 3455-3482.

Reinelt, J., 2009. Frictional Contact of Elastomer Materials on Rough Rigid Surfaces (Ph.D. thesis). Leibniz Universität Hannover, Hannover, Germany.

Ronsin, O., Coeyrehourcq, K.L., 2001. State, rate and temperature-dependent sliding friction of elastomers. Proc. R. Soc. Lond. A 457, $1277-1294$.

Sadowski, P., Stupkiewicz, S., 2010. Combined effect of friction and macroscopic deformation on asperity flattening. Tribol. Int. 43, 1735-1741.

Salti, B., Laraqi, N., 1999. 3-D numerical modeling of heat transfer between two sliding bodies: temperature and thermal contact resistance. Int. J. Heat Mass Transf. 42, 2363-2374.

Schallamach, A., 1953. The velocity and temperature dependence of rubber friction. Proc. Phys. Soc. B 66, $386-392$.

Shen, L., Glassmaker, N.J., Jagota, A., Hui, C.-Y., 2008. Strongly enhanced static friction using a film-terminated fibrillar interface. Soft Matter 8, 618-625.

Simo, J.C., 1987. On a fully three-dimensional finite-strain viscoelastic damage model formulation and computational aspects. Comput. Methods Appl. Mech. Eng. 60, 153-173.

Simo, J.C., 1998. Numerical analysis and simulation of plasticity. In: Ciarlet, P.G., Lions, J.L. (Eds.), Handbook of Numerical Analysis, vol. IV. Elsevier, Amsterdam.

Strömberg, N., Johansson, L., Klarbring, A., 1996. Derivation and analysis of a generalized standard model for contact, friction and wear. Int. J. Solids Struct. 33, 1817-1836.

Stupkiewicz, S., 2007. Micromechanics of Contact and Interface Layers. Springer, Berlin, Heidelberg, New York.

Stupkiewicz, S., Lewandowski, M.J., Lengiewicz, J., 2014. Micromechanical analysis of friction anisotropy in rough elastic contacts. Int. J. Solids Struct. 51, $3931-3943$

Suwannachit, A., Nackenhorst, U., Chiarello, R., 2012. Stabilized numerical solution for transient dynamic contact of inelastic solids on rough surfaces. Comput. Mech. 49, 769-788.

Temizer, İ., 2013. A mixed formulation of mortar-based contact with friction. Comput. Methods Appl. Mech. Eng. 255, $183-195$.

Temizer, İ., 2014a. Multiscale thermomechanical contact: computational homogenization with isogeometric analysis. Int. J. Numer. Methods Eng. 97, 582-607.

Temizer, İ., 2014b. Computational homogenization of soft matter friction: isogeometric framework and elastic boundary layers. Int. J. Numer. Methods Eng. 100, 953-981.

Temizer, İ., Wriggers, P., 2008. A multiscale contact homogenization technique for the modeling of third bodies in the contact interface. Comput. Methods Appl. Mech. Eng. 198, 377-396.

Temizer, İ, Wriggers, P., 2010a. Inelastic analysis of granular interfaces via computational contact homogenization. Int. J. Numer. Methods Eng. 84, 883-915.

Temizer, İ., Wriggers, P., 2010b. Thermal contact conductance characterization via computational contact homogenization: a finite deformation theory framework. Int. J. Numer. Methods Eng. 83, 27-58.

Temizer, İ., Wriggers, P., 2011. Homogenization in finite thermoelasticity. J. Mech. Phys. Solids 59, $344-372$.

Uchidate, M., Yanagi, K., Yoshida, I., Shimizu, T., Iwabuchi, A., 2011. Generation of 3D random topography datasets with periodic boundaries for surface metrology algorithms and measurement standards. Wear 271, 565-570.

van Kuilenburg, J., Masen, M.A., Groenendijk, M.N.W., Bana, V., van der Heide, E., 2012. An experimental study on the relation between surface texture and tactile friction. Tribol. Int. 48, 15-21.

Ward, I.M., Sweeney, J., 2004. The Mechanical Properties of Solids Polymers, 2nd ed. Wiley, Chichester.

Woodhouse, J., Wang, S.K., 2011. The frequency response of dynamic friction: model comparisons. J. Mech. Phys. Solids 59, 2294-2306.

Wriggers, P., 2006. Computational Contact Mechanics, 2nd ed. Springer, Berlin, Heidelberg, New York.

Wriggers, P., Reinelt, J., 2009. Multi-scale approach for frictional contact of elastomers on rough rigid surfaces. Comput. Methods Appl. Mech. Eng. 198, 1996-2008.

Yu, Q., Fish, J., 2002. Multiscale asymptotic homogenization for multiphysics problems with multiple spatial and temporal scales: a coupled thermoviscoelastic example problem. Int. J. Solids Struct. 39, 6429-6452. 